\title{
Enhanced upward heat transport at deep submesoscale ocean fronts
}

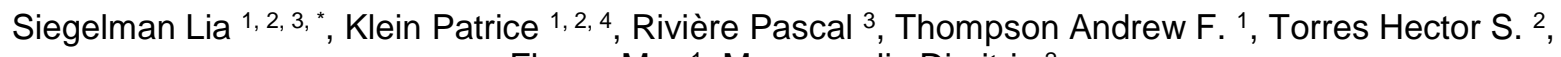
Flexas Mar ${ }^{1}$, Menemenlis Dimitris ${ }^{2}$

${ }^{1}$ Environmental Science and Engineering, California Institute of Technology, Pasadena, CA, USA

2 Jet Propulsion Laboratory, California Institute of Technology, Pasadena, CA, USA

3 Université de Brest, CNRS, IRD, Ifremer, LEMAR, Plouzané, France

${ }^{4}$ Université de Brest, CNRS, IRD, Ifremer, LOPS, Plouzané, France

*Corresponding author : Lia Siegelman, email address : Isiegelman@caltech.edu

\begin{abstract}
:
The ocean is the largest solar energy collector on Earth. The amount of heat it can store is modulated by its complex circulation, which spans a broad range of spatial scales, from metres to thousands of kilometres. In the classical paradigm, fine oceanic scales, less than $20 \mathrm{~km}$ in size, are thought to drive a significant downward heat transport from the surface to the ocean interior, which increases oceanic heat uptake. Here we use a combination of satellite and in situ observations in the Antarctic Circumpolar Current to diagnose oceanic vertical heat transport. The results explicitly demonstrate how deep-reaching submesoscale fronts, with a size smaller than $20 \mathrm{~km}$, are generated by mesoscale eddies of size 50-300 $\mathrm{km}$. In contrast to the classical paradigm, these submesoscale fronts are shown to drive an anomalous upward heat transport from the ocean interior back to the surface that is larger than other contributions to vertical heat transport and of comparable magnitude to air-sea fluxes. This effect can remarkably alter the oceanic heat uptake and will be strongest in eddy-rich regions, such as the Antarctic Circumpolar Current, the Kuroshio Extension and the Gulf Stream, all of which are key players in the climate system.
\end{abstract}


Mesoscale eddies are intensified in energetic areas such as the Antarctic Circumpolar Current (ACC), Kuroshio Extension and Gulf Stream ${ }^{4}$. They are known to drive the production of submesoscale fronts $(<20 \mathrm{~km})$, i.e. regions that separate waters of different densities ${ }^{5-9}$, ubiquitous on satellite images of ocean color (Fig. 1). To date, submesoscale fronts are mainly thought to be confined to the ocean surface mixed-layer $(\sim 100 \mathrm{~m} \mathrm{deep})^{8,9}$. This is because, in the classical paradigm, motions below the mixed-layer are broadly assumed to be in geostrophic balance (a balance between Coriolis and horizontal pressure forces), preventing the formation of strong density gradients ${ }^{10}$. As a consequence, fine scales below the mixed-layer are still principally associated with diffusive processes that drive downward heat transport. However, a handful of studies suggest that submesoscale fronts associated with upward heat transport may also penetrate below the mixed-laye ${ }^{11-14}$. Yet, these studies do not explain the formation mechanism of these submesoscale fronts because they are confined to small domains $(<50 \mathrm{~km})$. Observational evidence over domains sufficiently large to capture multiple mesoscale eddies and their associated submesoscale fronts are needed for the closure of oceanic vertical heat transport. Such measurements are still lacking due to the fact that capturing fine spatial and temporal scales over extended domains lie at the edge of our observational capabilities.

Here, we meet this observational challenge by analyzing a unique dataset collected by an instrumented elephant seal in the ACC. The seal data reveal the presence of numerous deep-reaching submesoscale fronts that extend well below the mixed-layer and are principally found on eddies' edges. We then develop a pioneering methodology, combining satellite and seal observations, to retrieve a 3-D synoptic view of ocean dynamics from meso- to submesoscale. Results explicitly demonstrate that deep-reaching submesocale fronts are generated by the strain field associated with co-interacting mesoscale eddies. By invoking properties of mesoscale turbulence, we explain why deep-reaching fronts, counter-intuitively, drive enhanced vertical heat transport below 
the surface mixed-layer that is directed upward, which is also supported by a numerical model analysis (see Supplementary Information). Associated vertical heat transport is larger than the mean flow and eddy contributions ${ }^{2}$, and of similar magnitude to air-sea fluxes ${ }^{15}$. This effect is argued to crucially limit oceanic heat uptake and therefore to alter the ocean heat storage capacity.

\section{Frontal region sampled by elephant seal and satellite}

Measurements were collected by a southern elephant seal (Mirounga leonina) in the Kerguelen area (Indian sector of the Southern Ocean, Fig. 1) during the austral spring and summer. The dataset has a horizontal resolution of $1 \mathrm{~km}$, a vertical resolution of $1 \mathrm{~m}$ and extends from the surface down to $400 \mathrm{~m}$ in the ocean interior. Over a period of more than $3 \mathrm{months}$ and a distance exceeding $5000 \mathrm{~km}$, the seal continuously recorded temperature, conductivity and pressure, from which buoyancy $b$ (of opposite sign to density) is derived (see Methods). An example of the buoyancy field is shown in Fig. 2a-c. In addition, satellite altimeter observations supply daily horizontal fields of Sea Surface Height (SSH), a proxy of surface pressure, with an effective resolution sufficient to resolve mesoscale eddies with a size of 50 to $100 \mathrm{~km}$.

The area sampled by the seal from km 1000 to 2300 (in red in Fig. 1), i.e., from 11/02-24/2014, is rich in eddies and lies within the energetic $\mathrm{ACC}^{16}$ as illustrated by the SSH contours in Fig. 1. The seal crossed numerous co-interacting cyclonic (low SSH) and anti-cyclonic (high SSH) mesoscale eddies. Geostrophic currents derived from SSH reach values of $1 \mathrm{~m} / \mathrm{s}$ around the eddy edges (Fig. 2a), similar to those found in the Gulf Stream and the Kuroshio Extension, the two other most energetic ocean currents $^{4}$. This area is thus representative of the flow field encountered within eddy-rich regions of the world oceans, and will receive particular emphasis in this study. The other areas crossed by the seal (Fig. 1) are much less energetic (Extended Data Fig. 1).

The comparison between satellite SSH and buoyancy anomalies sampled by the seal in the turbulent area (Figs. 2a-c and $\mathrm{S} 1, \mathrm{a}-\mathrm{c}$ ) highlights the expected vertical structure of the mesoscale eddies ${ }^{10}$ : buoyancy anomalies are positive and bowl-shaped in anticyclonic eddies (high SSH) and negative and reversed bowl-shaped in cyclonic eddies (low SSH, Figs. 2a-c and Extended Data Fig. 1a-c). The combination of satellite observations of SSH and seal measurements of buoyancy provides a synoptic 3-D view of the flow field encountered by the seal, and in particular of the eddy field, that can extend down to depths of at least 400 $\mathrm{m}$. We refer the reader to the Supplementary Information for a more detailed analysis of the consistency at mesoscale between these two independent datasets.

Frontal structures, or buoyancy fronts, are identified by the along-track derivative of buoyancy and are defined as $b_{s}=\partial b / \partial s$, with $s$ the curvilinear abscissa, i.e. the along-track direction. The fronts, shown in Fig. $2 \mathrm{~d}$, have a width between 5 and $20 \mathrm{~km}$ and are thus submesoscale features. Indeed, the Rossby radius of deformation in the Kerguelen area is of $\sim 15 \mathrm{~km}{ }^{17}$, which corresponds to an eddy radius of $\sim 50 \mathrm{~km}^{17,18}$, consistent with the SSH observations (Fig. 2a). These fronts are well resolved by the seal's 1-km horizontal resolution measurements. They are more numerous below the mixed-layer and extend down to at least $400 \mathrm{~m}$. Large buoyancy gradients are preferentially found at the edges of the mesoscale eddies and in-between them. Buoyancy gradient magnitudes reach values larger than $5 \times 10^{-7} \mathrm{~s}^{-2}$. The root mean square of the lateral gradient of buoyancy, used as an indicator of the gradient magnitude, is larger than $0.5 \times 10^{-7} \mathrm{~s}^{-2}$, regardless of depth (Extended Data Fig. 2a). Compared to the few other existing submesoscale-resolving datasets ${ }^{19-21}$, these values are of the same order of magnitude, highlighting the important frontal activity of the area.

\section{Frontal dynamics in the ocean interior}

Fig. 3 illustrates how the production of horizontal gradients of buoyancy is driven by a pure strain field, which corresponds to the hyperbolic regions in-between co-interacting eddies ${ }^{5}$, as can be seen in Fig. 1. In the schematic Fig. 3a, the strain field (black arrows) stretches a tracer patch in the y-direction and compresses it in the x-direction. This leads to the formation of strong horizontal gradients of buoyancy, or fronts, at submesoscale that are associated with a growth rate related to the strain rate $u_{x}=\frac{\partial u}{\partial x}$ (Fig. 3b).

To assess the relevance of this mechanism in the Kerguelen area, we analyze the background strain field in relation to the observed buoyancy gradients. We use daily Finite Size Lyapunov Exponents (FSLE) ${ }^{22}$ computed from satellite-derived geostrophic velocities to characterize the strain field properties (see Methods). FSLE indicate both the orientation and time scale (colorbar in Fig. 4a) of the stretching and compression induced by the strain field (respectively the red and blue curves in Figs. 3a and 4a). As illustrated in Fig. 3a, we expect tracer patch, or equivalently buoyancy anomalies, to be aligned with stretching FSLE $^{22}$. The spatial distribution of FSLE (Fig. 4a and Extended Data Fig. 3) confirms that regions in-between eddies and on 
eddy edges are prone to the formation of strong horizontal buoyancy gradients. However, the seal's trajectory is often oblique rather than perpendicular to the buoyancy fronts, which may lead to an underestimation of the front's magnitude. Therefore, to correct for the seal's orientation with respect to the FSLE it encounters, buoyancy gradients are first normalized by the angle between the seal's trajectory and the FSLE direction (see Methods). Note that normalized buoyancy gradients are now referred to as $b_{x}$, where the subscript $x$ refers to the direction normal to the stretching FSLE (Fig. 3a). We then compare the along-track time series of stretching FSLE estimated along the seal's path with the normalized buoyancy gradients at $300 \mathrm{~m}$ (comparison with buoyancy gradients at different depths below the mixed-layer produces similar results). There is good agreement between FSLE and normalized buoyancy gradients at $300 \mathrm{~m}$ (Fig. 4 and Extended Data Fig. 4a), as well as a consistent relationship linking the two (Fig. 4c). The two time-series are strongly intermittent. Peaks of FSLE and buoyancy gradients are often co-located (Fig. 4a-b and Extended Data Fig. 4a). Note that similar results are obtained with non-normalized gradients, which demonstrates that no bias is added via the normalization. Combined together, these two datasets provide observational evidence of how an eddy strain field, diagnosed at the sea surface, drives strong buoyancy gradients at submesoscale in the ocean interior.

Comparison between panels $\mathrm{c}$ and d in Fig. 2 further reveals that, whereas mesoscale buoyancy anomalies are slanted on the vertical, as evidenced by the bowl-shaped structures of buoyancy (panel c), buoyancy fronts at submesoscale are almost vertical (panel d). This density slope property is related to the dynamical regime that drives these structures and can be quantified by the non-dimensional Richardson number $R i$ (see Methods). The small Ri-values observed in Fig. $2 \mathrm{~d}$ suggest an energetic ageostrophic regime associated with intense vertical currents. The emergence of these vertical currents, $w$, counter-balances the formation of sharp submesoscale fronts generated by the ambient strain field. This mechanism is referred to as frontogenesis ${ }^{23}$ and is sketched in Fig. 3b. It leads to an equilibrium that is captured by the classical omega equation, which involves the vertical current field $w$, the strain field $u_{x}$, and the lateral gradient of buoyancy field $b_{x}$. Here, we diagnose the vertical currents $w$ by solving the 2-D (x,z) quasi-geostrophic version of the omega equation ${ }^{23}$ (see Methods), with $b_{x}$ obtained from seal observations and $u_{x}$ estimated from stretching FSLE (Fig. 4). However, compared to the full omega equation, there is a tendency for the vertical currents diagnosed here to be underestimated by $\sim 1.4$ (see Methods).

The vertical section of $w$ shown in Fig. $5 \mathrm{~b}$ reveals positive and negative $w$-values with large magnitudes of up to $100 \mathrm{~m} / \mathrm{day}$, i.e. almost an order of magnitude greater than what is attributed to mesoscale eddies alone ${ }^{24}$. Vertical currents have a width of 5 to $10 \mathrm{~km}$. They are intensified in the ocean interior, below the mixed-layer down to at least $400 \mathrm{~m}$, and do not necessarily penetrate into the mixed-layer. The continuous vertical extent of these vertical currents highlights the crucial role played by deep-reaching submesoscale fronts in connecting the ocean interior to the surface. The $w$-field is characterized by the same intermittency present in the FSLE and buoyancy gradient fields (Figs. 2d, 4c, 5b and Extended Data Fig. 4b). Large $w$-magnitudes are co-located with strong buoyancy gradients and intense FSLE, and are therefore mostly found on the edges of, and in-between, eddies.

The analysis of the vertical currents presented here is supported by a comparison with a realistic regional numerical model with a 1.5-km horizontal resolution (see Supplementary Information for more details on the model). The values of $w$ derived from the observations are comparable to, although smaller than, what is obtained in the model (Extended Data Fig. 5a). Overall, these results suggest that the vertical pathway provided by deep ocean fronts in spring and summer is likely a generic mechanism throughout the ocean. This deep-reaching vertical pathway has important consequences for the vertical transfer of heat between the ocean interior and the surface mixed-layer, as explored in the next section.

\section{Vertical heat transport at deep submesoscale fronts}

Oceanic heat transport is estimated from temperature and vertical velocity anomalies, from the surface down to $400 \mathrm{~m}$ depth (Fig. 5a-b). A vertical section of this transport (see Methods for the calculation) is shown in Fig. 5c, where a positive (negative) value indicates an upward (downward) heat transport. Positive values result from frontogenesis processes, i.e the production of fronts, as illustrated in Fig. 3b. On the other hand, negative values arise from frontolysis processes, i.e. the destruction of fronts (see Supplementary Information). Heat transport is strongly enhanced at the location of submesoscale fronts generated by the background strain field and has a local amplitude that reaches $2000 \mathrm{~W} / \mathrm{m}^{2}$ below the surface mixed-layer, and extending down to at least $400 \mathrm{~m}$, consistent with the high-resolution regional model (Extended Data Figs. 5b and 6) and, surprisingly, of the same order of magnitude as instantaneous air-sea heat fluxes ${ }^{19}$.

We now explore the contribution of fine oceanic scales to the domain-averaged vertical heat transport. Averaged vertical heat transport within the eddy-rich area of the ACC sampled by the seal (km 1000 to 2000, in red in Fig. 1) is directed upward, i.e. from the ocean interior back to the surface (Fig. 5f). This direction is strikingly opposite to the one induced by the diffusive processes used in the classical paradigm. However, this result can be understood in terms of the direct cascade 
of potential energy, a well-known property of mesoscale eddy turbulence ${ }^{25}$. The direct cascade implies that frontogenesis statistically dominates frontolysis, and thus that the net vertical heat transport associated to submesoscale fronts is positive (see Supplementary Information), which is also striking in the model outputs (Extended Data Fig. 6). Furthermore, the heat transport magnitude reaches an averaged value of $\sim 100 \mathrm{~W} / \mathrm{m}^{2}$ at $200 \mathrm{~m}$ (Fig. 5f). Remarkably, this value is an order of magnitude larger than that associated with mesoscale eddies alone ${ }^{26}$, which is, along with the mean flow, traditionally thought to be the main contributor to vertical heat transport ${ }^{2}$. It is also of comparable magnitude to domain-averaged air-sea heat fluxes ${ }^{15}$, illustrating a potential coherent pathway linking the ocean interior to the atmosphere. Note that the percentage of seal dives decreases with depth (gray line in Fig. 5f), from 100 to $40 \%$ between 200 and $400 \mathrm{~m}$. This is likely to account for a part of the RMS decrease (stagnation) of temperature (velocity) anomalies below $200 \mathrm{~m}$, as one would have expected ${ }^{5}$ (Fig. $5 \mathrm{~d}$,e). As a consequence, the already unexpected strong heat transport (Fig. 5c,f) is likely to be underestimated because of it, in addition to the conservative estimate of the vertical currents discussed in the previous section. Indeed, the domain-integrated heat transport from the high-resolution numerical simulation yields a similar - although stronger - positive vertical heat transport (Extended Data Fig. 7) with, for instance, a value of $\sim 140 \mathrm{~W} / \mathrm{m}^{2}$ at $200 \mathrm{~m}$. As such, these results strongly contrast with the classical paradigm based on diffusive heat transport, as they emphasize the existence of intense and upward heat transport in the ocean interior well below the ocean surface mixed-layer that are preferentially localized in strain dominated areas (Extended Data Fig. 6).

The data presented here provide the first observational evidence of large and anomalous, i.e. upward, heat transport associated with deep-reaching submesoscale fronts in an eddy-rich area of the world ocean. The observations, supported by a high-resolution regional model (see Supplementary Information), highlight the crucial role played by submesoscale frontal dynamics, in the ocean interior well below the ocean surface mixed-layer, for oceanic heat transport.

In summary, The deep-reaching frontal dynamics and its associated large positive vertical heat transport, observed here in numerous sharp fronts of the ACC, are driven by mesoscale eddies and are likely to occur widely in the ocean, such as in the eddy-rich Gulf Stream and Kuroshio Extension, all of which are key players in the climate system. It potentially plays an important role by, for instance, exacerbating restratification processes as warm (cold) waters become warmer (colder). Furthermore, a first order estimation indicates these deep ocean fronts lead to an additional increase of the sea surface temperature ranging between $\sim 0.2$ and $\sim 0.6^{\circ} \mathrm{C}$ within a month (see Methods for the calculation and caveats), illustrating the potential impact of deep-reaching ocean fronts on air-sea fluxes and how they can substantially limit oceanic heat uptake from the atmosphere.

These observational results suggest revisiting current estimates of the Earth's heat budget and stress the need to account for small-scale physics, not only within, but also below the ocean surface mixed-layer, in the prediction of future climate states. Inaccurate representation of these physics could considerably underestimate the amount of heat transferred from the ocean interior back to the surface and, as a consequence, potentially overestimate the amount of heat the ocean can absorb. Finally, these results may have a far greater scope as the evidence for intense vertical currents associated to the deep-reaching ocean fronts presented here also provide an efficient pathway for the transport of chemical and biological tracers, with potential major implications for biogeochemical systems.

\section{Methods}

\section{Sea Surface Height and geostrophic currents}

The Sea Surface Height (SSH) used to retrieve the mesoscale dynamics of the Kerguelen area $\left(67-91^{\circ} \mathrm{E}, 48-58^{\circ} \mathrm{S}\right)$ is composed of the Mean Dynamic Topography CNES/CLS $2015^{27}$ and the Sea Level Anomaly maps produced by the DUACS processing chain merging the multi-altimeter along-track data. This specific regional dataset was produced in the context of the DUACSHR project aiming at increasing the resolution of altimetry maps by applying recently developed methods such as dynamic interpolation ${ }^{28}$. The period from October 2014 to January 2015 benefits from an extremely favorable satellite coverage of four altimeters: AltiKa, Jason-2, Cryosat-2 and HY-2, allowing a very good spatial and temporal sampling. The temporal resolution is daily and, as estimated in Ballarotta et al. (personal communication), the spatial effective resolution of the gridded anomalies approaches $40 \mathrm{~km}$ in wavelength. Using the geostrophic approximation, geostrophic surface currents $(\mathrm{u}, \mathrm{v})$ are derived from SSH following ${ }^{10}$ :

$$
u=\frac{g}{f} \frac{\partial S S H}{\partial x}, v=-\frac{g}{f} \frac{\partial S S H}{\partial y},
$$

where $g$ is gravity and $f$ the Coriolis parameter. 
Instead of using a direct estimate of the strain rate (i.e., du/dx calculated directly from SSH, Fig. 3) that only describes the buoyancy gradient's growth rate, we use Finite Size Lyapunov Exponents (FSLE). Indeed, FSLE have the advantage of exploiting both the spatial and temporal variability of the velocity field deduced from SSH, and consequently provide information about both the growth rate and orientation of elongated buoyancy gradients ${ }^{29,30}$. FSLE is a Lagrangian diagnostic that measures the separation of close initial particles embedded in a given flow field. The separation's growth rate is defined as

$$
\lambda\left(d_{0}, d_{f}\right)=\frac{1}{\tau} \log \left(\frac{d_{f}}{d_{0}}\right),
$$

where $d_{0}\left(d_{f}\right)$ is the initial (final) separation distance and $\tau$ the first time at which a separation $d_{f}$ is reached. FSLE has the dimension of time ${ }^{-1}$. FSLE is particularly suited to diagnose the properties of a strain field. Positive (negative) FSLE indicate that patches of particles are being stretched (compressed) in a given direction determined by the background strain field. As such, large positive (negative) FSLE values indicate regions of strong stretching (compression) (see Fig. 3a and Supplementary Information for more details). Here, positive (negative) FSLE are computed backward (forward) in time ${ }^{22}$.

88 daily maps of altimetry-based FSLEs were computed for the Kerguelen area following d'Ovidio et al. (2004) ${ }^{22}$ and using the geostrophic velocities derived from SSH. Parameters were chosen close to d'Ovidio et al. $(2004)^{22}$ with $d_{0}=0.04^{\circ}$ and $d_{f}=1^{\circ}$, i.e. a final separation distances of about $110 \mathrm{~km}$.

An along-track time series of stretching (i.e. positive) FSLE was extracted along the seal's path in order to capture the stretching direction and intensity of the strain field. A subsequent 5-km window moving average was applied to remove the strong intermittency present in the raw data (Fig. 4 and Extended Data Fig. 4a). Stretching FSLEs are then compared to lateral gradients of buoyancy sampled by the seal.

\section{Southern Elephant Seal measurements}

A newly released in situ dataset collected in the austral summer by a female southern elephant seal during her three-month post-breeding trip (20 October 2014 to 16 January 2015) east of the Kerguelen Islands (Indian sector of the Southern Ocean, see Fig. 1) is analyzed. Compared to previous tags mounted on elephant seals, this one records every single dive realised by the animal (>80 dives/day) at high-resolution as opposed to 2 to 5 dives/day for previous tags. The seal is localized through the Argos satellite system and is equipped with sensors recording conductivity, temperature and pressure (CTD-Satellite Relay Data Logger) at a continuous frequency of $0.5 \mathrm{~Hz}$. Only the ascending phase of a dive is used because it is more uniform in speed and direction compared to the descent when the seal dives sinuously to forage ${ }^{31}$. The dataset is comprised of 6333 dives, which corresponds to a cumulative length of $5270 \mathrm{~km}$ with a median spacing between two dives of $700 \mathrm{~m}$ (Extended Data Fig. 8a). Dives, that can be as deep as 500 to $1000 \mathrm{~m}$, last less than 25 minutes and are separated by intervals of a few minutes where the seal breathes but does not transit. More than $80 \%$ of the dives reach a depth of at least $200 \mathrm{~m}, 50 \%$ reach $300 \mathrm{~m}$ and $35 \% 400 \mathrm{~m}$ or deeper.

To ensure a better accuracy of the temperature and conductivity-derived salinity data, two additional steps are applied: first a thermal cell effect correction is applied to the temperature and conductivity fields. A density inversion removal algorithm, which seeks the minimum adjustment of the salinity profile to achieve neutral stability is subsequently applied to the salinity field. The accuracy of the final post-processed data is of $\pm 0.02^{\circ} \mathrm{C}$ for temperature and $\pm 0.03 \mathrm{~g} / \mathrm{kg}$ for salinity. More details on the post-processing method and final data accuracy can be found in Siegelman et al. (2019) ${ }^{31}$. Potential density is calculated from corrected conservative temperature and absolute salinity with the TEOS-10 equation ${ }^{32}$.

The animal in this study was handled in accordance with the Institut polaire francais Paul-Emile Victor (IPEV) ethical and Polar Environment Committees guidelines as part of the SNO-MEMO and IPEV program 109 (PI. H. Weimerskirch). The experimental protocols were approved by the Ethics Committee of IPEV and Polar Environment Committees.

\section{Buoyancy}

Along-track time series of buoyancy, $b=g\left(1-\rho / \rho_{0}\right)$, where $\mathrm{g}$ is gravity, $\rho$ is potential density, and $\rho_{0}=1025 \mathrm{~kg} \mathrm{~m}^{-3}$ is a reference density reveals variability covering both meso- and submesoscales. For the analysis, in particular the calculation of lateral buoyancy gradients $b_{s}=\partial b / \partial s$ with $s$ the curvilinear abscissa, buoyancy was first linearly interpolated along the seal's path onto a regular grid of $100 \mathrm{~m}$ resolution, corresponding to the shortest along-track distance between two dives (Extended Data Fig. 6). A moving average with a $1 \mathrm{~km}$ window was then applied such that the final dataset has a horizontal resolution of 1 $\mathrm{km}$ and a vertical resolution of $1 \mathrm{~m}$. Buoyancy anomalies are resolved by multiple vertical profiles, such that the structures are 
not related to aliasing of the along-track data. Surface buoyancy anomalies in Fig. 2a,b is defined by the time series of the mean buoyancy from 15 to $50 \mathrm{~m}$ minus its mean value calculated from 15 to $50 \mathrm{~m}$ along the entire trajectory.

\section{Mixed layer depth}

The mixed layer depth (MLD) is defined as the level of a $0.03 \mathrm{~kg} \mathrm{~m}^{-3}$ density increase from $15 \mathrm{~m}$ depth.

\section{Normalization of buoyancy gradients}

Since the seal's trajectory is more often oblique to the stretching FSLE it crosses rather than perpendicular to them (Extended Data Fig. 8b) and buoyancy fronts are assumed to be aligned with stretching FSLE, buoyancy gradients sampled by the seal need to be corrected in order to account for the seal's orientation with respect to the FSLE it encounters. To do so, buoyancy gradients are divided by the sine of the angle $\theta$ between the seal's trajectory and the FSLE direction. More precisely, $\theta$ is the angle between the FSLE eigenvector and the seal's path. To focus on the regions prone to the formation of submesoscales, only $b_{s}$ associated with large FSLE $\left(>0.15\right.$ day $\left.^{-1}\right)$ have been normalized and are then referred to as $b_{x}$. However, a sensitivity analysis (ranging from 0.1 to 0.3 day $^{-1}$, not shown here) to this threshold value leads to similar results.

\section{Link between strain, frontogenesis and vertical heat transport (Fig. 3)}

The ambient strain field in Fig. 3a acts to elongate the tracer patch in the stretching direction (y-direction) and to compress it in the $\mathrm{x}$-direction since the area of the tracer patch is conserved to leading order ${ }^{10}$. This increases the tracer gradient in the $\mathrm{x}$-direction. The orientation and time scale of the strain field can be captured by the Finite Size Lyapunov Exponents (FSLE, see main text and Methods). Red (blue) FSLE lines identify the horizontal stretching (compressing) direction. This mechanism is particularly relevant for the formation of submesoscale fronts, that are thus aligned with the stretching FSLE (red line).Fig. $3 \mathrm{~b}$ shows a schematic illustration of the frontogenesis process. The Fig. corresponds to a 3-D slice through a submesoscale front generated by a background mesoscale strain field (curved black arrows) like the one in a). Thin black lines are isotherms. Straight black arrows correspond to the vertical velocities that develops in response to the front intensification. Warmer (or equivalently lighter) fluid (light red) is on the right of the Fig. and colder (or equivalently heavier) fluid (light blue) is on the left. As a consequence, the vertical heat transport associated to frontogenesis is directed upward because of the positive correlation between temperature and vertical velocity anomalies.

\section{Richardson number}

The non-dimensional Richardson number $R i$, estimated from the seal measurements, is defined as $R i \equiv f^{2} N^{2} / b_{x}^{2}$, with $N^{2}=$ $\partial b / \partial z$ the Brunt-Väissälä frequency and $b_{x}=\partial b / \partial x$ the along-track lateral gradient of buoyancy. $R i$ characterizes the dynamical regime and can be interpreted as the steepness of the isopycnal slopes relatively to $N / f$, since $R i=\frac{f^{2}}{N^{2}}\left(\frac{\partial b / \partial z}{\partial b / \partial x}\right)^{2}=\frac{f^{2}}{N^{2}}\left(\frac{\partial x}{\partial z}\right)^{2}$. Thus, for $R i>>1$, which corresponds to the quasi-geostrophic regime ${ }^{33,34}$, the steepness of the isopycnal slope, $\frac{\partial b / \partial x}{\partial b / \partial z}$, is small. For $R i$ close to one (i.e. $R i \leq 4)$, which corresponds to the ageostrophic regime ${ }^{33,34}$, the steepness of the isopycnal slope, $\frac{\partial b / \partial x}{\partial b / \partial z}$, is large. Seal observations suggest a strong ageostrophic regime since instances of strong $b_{x},\left|b_{x}\right| \geq 2.5 \times 10^{-7} \mathrm{~s}^{-2}$, coincide with $R i<2$ (Extended Data Fig. 2b). Small $R i$-values $(R i<2)$ indicate an ageostrophic regime where vertical currents are large ${ }^{33,34}$. Instances of strong $b_{x},\left|b_{x}\right| \geq 2.5 \times 10^{-7} \mathrm{~s}^{-2}$, coincide with $R i<2$ (Fig. 2 and Extended Data Fig. 2b).

\section{Vertical velocities}

Classical methods to diagnose vertical velocities are based on the Q-vector version of the omega equation ${ }^{23,35}$. In this study, we use the quasi-geostrophic (QG) version of the omega equation (see the Supplementary Information section for a discussion about this choice). Buoyancy fronts are assumed to be elongated along a stretching direction (red FSLE in Fig. 3a) such that the along-front gradient of buoyancy in the $y$-direction is negligible with respect to the cross-front one (x-direction). Thus, we consider the 2-D version $(\mathrm{x}, \mathrm{z})$ of the $\mathrm{QG}$ omega equation. This equation assumes that the trajectory is normal to the front, i.e. that the front is embedded in a pure strain field, which is achieved through the buoyancy gradients normalization mentioned above. This equation reads :

$$
N^{2} w_{x x}+f^{2} w_{z z}=-2\left(u_{x} b_{x}\right)_{x},
$$

where subscripts indicate derivatives. $u_{x}$ is estimated from stretching FSLE derived from satellite altimetry and $b_{x}, N^{2}$ and $f^{2}$ from the seal's measurements.

Equation (3) is solved using the flexible framework for spectrally solving differential equations provided by dedalus ${ }^{36}$. 
Note that when the Richardson number is close to one, equation (3) underestimates vertical velocities. When $R i \sim 2$, the magnitude of this underestimation is of $\sim 0.7$, implying that the vertical velocities $w$ diagnosed at sharp fronts should be closer to $1.4 \times w$ (see Supplementary Information for more details).

Note also that this study focuses on strain dominated regions. However, in other regions, for instance, within mesoscale eddies, the strain rate is weaker and its impact on the formation of horizontal gradients is counterbalanced by the impact of the relative vorticity, which leads to the formation of weaker gradients of buoyancy. In such regions, even though the 2-D version of the omega equation is no longer appropriate and a 3-D version needs to be used, the resulting vertical velocities are 7.5 times weaker than the ones associated to submesoscale fronts in strain dominated regions ${ }^{5}$, like the ones considered in the present study.

\section{Vertical heat transport}

The vertical heat transport is defined as $\rho C_{p} w^{\prime} T^{\prime}$, where $C_{p}=3985 \mathrm{~J} \mathrm{~kg}^{-1} \mathrm{~K}^{-1}$ is the specific heat capacity of seawater, $\rho=$ $1025 \mathrm{~kg} \mathrm{~m}^{-3}$ is the density of the fluid, $w^{\prime}$ and $T^{\prime}$ the vertical velocity and temperature anomalies, respectively.

\section{Impact of deep-reaching ocean fronts on the mixed-layer temperature}

Impact of deep-reaching ocean fronts on the mixed-layer temperature, and therefore the sea surface temperature, is derived from a thermodynamic equation that captures the evolution of the mixed-layer temperature $T$. This approach has already been used and validated at leading $\operatorname{order}^{26}$. The equation, integrated over the mixed-layer depth, reads:

$$
C \frac{d T}{d t}=S-\lambda T,
$$

where $C=\rho C_{p} H$ is the total heat capacity of the mixed-layer. $H$ is the mixed-layer depth, $S$ is the heat transport at the base of the mixed-layer due to deep-reaching fronts, and $-\lambda T$ is the negative feedback from air-sea heat fluxes. From equation (4), positive $S$ causes a higher temperature $T$, and hence a larger upward air-sea heat exchange $\lambda T$.

A sensitivity analysis is carried out to assess the range of the mean mixed-layer temperature change due to submesoscales over a period of one month, which corresponds to the time spent by the seal in the turbulent region and the time span of the high-resolution model. We set $S$ to vary between 50 and $100 \mathrm{~W} / \mathrm{m}^{2}$, as obtained from both the observational (Fig. 5f) and model (Extended Data Fig. 7) results, $\lambda$ to vary between 15 and $25 \mathrm{~W} \mathrm{~m}^{-2} \mathrm{~K}^{-1}$, according to Vallis (2012) ${ }^{37}$, and the mixed-layer $H$ to vary between 100 and $200 \mathrm{~m}$, implying that $C$ varies between $\sim 4 \times 10^{8}$ and $\sim 8 \times 10^{8} \mathrm{~J} \mathrm{~m}^{-2} \mathrm{~K}^{-1}$. As a consequence, equation (4) integrated over one month indicates that the mean mixed-layer temperature increase ranges between $\sim 0.2$ and $\sim 0.6^{\circ} \mathrm{C}$.

Note this is a first order estimation that includes several caveats. In particular, this estimation does not take into account any subsequent atmospheric feedback on the ocean that may arise in response to these surface heat fluxes $(\lambda T)$. For instance, such feedback may include interactions between the windstress and SST anomalies at submesoscales ${ }^{38}$, which may limit the mean temperature increase.

\section{Data availability}

The marine mammal data were collected and made freely available by the International MEOP Consortium and the national programs that contribute to it, and is available at http://www.meop. net/database/meop-databases/ meop-sms-database-submesosc.html. The Ssalto/Duacs altimeter products were produced and distributed by the Copernicus Marine and Environment Monitoring Service with support from CNES, and is available at http://marine. copernicus.eu/services-portfolio/access-to-products/.

\section{References}

1. Munk, W. \& Wunsch, C. Abyssal recipes ii: Energetics of tidal and wind mixing. Deep. Sea Res. Part I: Oceanogr. Res. Pap. 45, 1977-2010 (1998).

2. Wolfe, C., Cessi, P., McClean, J. \& Maltrud, M. Vertical heat transport in eddying ocean models. Geophys. Res. Lett. 35, L23605 (2008).

3. Griffies, S. M. et al. Impacts on ocean heat from transient mesoscale eddies in a hierarchy of climate models. J. Clim. 28, 952-977 (2015). 
4. Ferrari, R. \& Wunsch, C. Ocean circulation kinetic energy: Reservoirs, sources, and sinks. Annu. Rev. Fluid Mech. 41, 253-282 (2009).

5. Klein, P. \& Lapeyre, G. The oceanic vertical pump induced by mesoscale and submesoscale turbulence. Annu. review marine science 1, 351-375 (2009).

6. Ferrari, R. A frontal challenge for climate models. Science 332, 316-317 (2011).

7. Mahadevan, A. The impact of submesoscale physics on primary productivity of plankton. Annu. review marine science $\mathbf{8}$, 161-184 (2016).

8. McWilliams, J. C. Submesoscale currents in the ocean. Proc. R. Soc. A 472, 20160117 (2016).

9. Lévy, M., Frank, P. J. \& K., S. S. The role of submesoscale currents in structuring marine ecosystems. Nat. Commun. DOI: 10.1038/s41467-018-07059-3 (2018).

10. Vallis, G. K. Atmospheric and oceanic fluid dynamics (Cambridge University Press, 2017).

11. Taylor, J. R. \& Ferrari, R. Ocean fronts trigger high latitude phytoplankton blooms. Geophys. Res. Lett. 38, 23 (2011).

12. Thomas, L. N., Taylor, J. R., Ferrari, R. \& Joyce, T. M. Symmetric instability in the gulfstream. Deep. Res. II 91, 96-110 (2013).

13. Bachman, S. D. \& Taylor, J. R. Modelling of partially-resolved oceanic symmetric instability. Ocean. Model. 82, 15-27 (2014).

14. Yu, X. et al. An annual cycle of submesoscale vertical flow and restratification in the upper ocean. J. Phys. Oceanogr. 49, 1439-1461 (2019).

15. Large, W. G. \& Yeager, S. The global climatology of an interannually varying air-sea flux data set. Clim. dynamics 33, 341-364 (2009).

16. Hogg, A. M. et al. Recent trends in the southern ocean eddy field. J. Geophys. Res. Ocean. 120, 257-267 (2015).

17. Chelton, D. B., DeSzoeke, R. A., Schlax, M. G., El Naggar, K. \& Siwertz, N. Geographical variability of the first baroclinic rossby radius of deformation. J. Phys. Oceanogr. 28, 433-460 (1998).

18. Chelton, D. B., Schlax, M. G., Freilich, M. H. \& Milliff, R. F. Satellite measurements reveal persistent small-scale features in ocean winds. science 303, 978-983 (2004).

19. Thompson, A. F. et al. Open-ocean submesoscale motions: A full seasonal cycle of mixed layer instabilities from gliders. J. Phys. Oceanogr. 46, 1285-1307 (2016).

20. du Plessis, M., Swart, S., Ansorge, I. J. \& Mahadevan, A. Submesoscale processes promote seasonal restratification in the subantarctic ocean. J. Geophys. Res. Ocean. 122, 2960-2975 (2017).

21. Viglione, G. A., Thompson, A. F., Flexas, M. M., Sprintall, J. \& Swart, S. Abrupt transitions in submesoscale structure in southern drake passage: glider observations and model results. J. Phys. Oceanogr. 48, 2011-2027 (2018).

22. d'Ovidio, F., Fernández, V., Hernández-García, E. \& López, C. Mixing structures in the mediterranean sea from finite-size lyapunov exponents. Geophys. Res. Lett. 31 (2004).

23. Hoskins, B. J., Draghici, I. \& Davies, H. C. A new look at the $\omega$-equation. Q. J. Royal Meteorol. Soc. 104, 31-38 (1978).

24. McGillicuddy Jr, D. J. Mechanisms of physical-biological-biogeochemical interaction at the oceanic mesoscale. Annu. Rev. Mar. Sci. 8, 125-159 (2016).

25. Salmon, R. Baroclinic instability and geostrophic turbulence. Geophys. \& Astrophys. Fluid Dyn. 15, 167-211 (1980).

26. Su, Z., Wang, J., Klein, P., Thompson, A. F. \& Menemenlis, D. Ocean submesoscales as a key component of the global heat budget. Nat. communications $\mathbf{9 ,} 775$ (2018).

27. Rio, M. H. et al. Improving the altimeter-derived surface currents using high-resolution sea surface temperature data: A feasability study based on model outputs. J. Atmospheric Ocean. Technol. 33, 2769-2784 (2016).

28. Ubelmann, C., Klein, P. \& Fu, L. L. Dynamic interpolation of sea surface height and potential applications for future high-resolution altimetry mapping. J. Atmospheric Ocean. Technol. 32, 177-184 (2015).

29. Waugh, D. W., Abraham, E. R. \& Bowen, M. M. Spatial variations of stirring in the surface ocean: A case study of the tasman sea. J. Phys. Oceanogr. 36, 526-542 (2006).

30. d'Ovidio, F., Isern-Fontanet, J., López, C., Hernández-García, E. \& García-Ladona, E. Comparison between eulerian diagnostics and finite-size lyapunov exponents computed from altimetry in the algerian basin. Deep. Sea Res. Part I: Oceanogr. Res. Pap. 56, 15-31 (2009). 
31. Siegelman, L. et al. Correction and accuracy of high-and low-resolution ctd data from animal-borne instruments. $J$. Atmospheric Ocean. Technol. 36, 745-760 (2019).

32. Intergovernmental-Oceanographic-Commission et al. The international thermodynamic equation of seawater-2010: calculation and use of thermodynamic properties.[includes corrections up to 31st october 2015]. UNESCO (2010).

33. Molemaker, J., McWilliams, J. \& Yavneh, I. Baroclinic instability and loss of balance. J. physical oceanography 35, 1505-1517 (2005).

34. Thomas, L. N., Tandon, A. \& Mahadevan, A. Submesoscale processes and dynamics. Ocean. modeling an Eddying Regime 177, 17-38 (2008).

35. Giordani, H., Prieur, L. \& Caniaux, G. Advanced insights into sources of vertical velocity in the ocean. Ocean. Dyn. 56, 513-524 (2006).

36. Burns, K. J., Vasil, G. M., Oishi, J. S., Lecoanet, D. \& Brown, B. Dedalus: Flexible framework for spectrally solving differential equations. Astrophys. Source Code Libr. (2016).

37. Vallis, G. K. Climate and the Oceans (Princeton University Press, 2012).

38. Foussard, A., Lapeyre, G. \& Plougonven, R. Response of surface wind divergence to mesoscale sst anomalies under different wind conditions. J. Atmospheric Sci. 76, 2065-2082 (2019).

\section{Corresponding author}

Correspondence and requests for materials should be addressed to Lia Siegelman, lsiegelman@caltech.edu.

\section{Acknowledgements}

Thanks to Kelvin Richards for his insightful comments, Francesco d'Ovidio for providing the code to compute FSLE and two anonymous reviewers whose comments improved the manuscript. This work was supported by the CNES-TOSCA project Elephant seals as Oceanographic Samplers of submesoscale features led by C. Guinet with support of the French Polar Institute (Progams 109 and 1201). This research was carried out, in part, at the Jet Propulsion Laboratory, California Institute of Technology, under a contract with the National Aeronautics and Space Administration (NASA). High-end computing resources for the numerical simulation were provided by the NASA Advanced Supercomputing Division at the Ames Research Center. This work was partly funded by the CNES (OSTST-OSIW) and the Laboratoire d'Excellence LabexMER (ANR-10-LABX-19). L.S. is a NASA-JVSRP affiliate and is supported by a joint CNES-Région Bretagne doctoral grant. P.K. is supported by the NASA-CNES SWOT mission and a NASA Senior NPP Fellowship. A.F.T. is supported by the David and Lucille Packard Foundation and NASA grant NNX16AG42G. M.F. is supported by NASA grant NNX15AG42G.

\section{Author contributions statement}

L.S. and P.K. conceived the experiments, analysed the results and wrote the manuscript. D.M. and H.S.T. ran the numerical simulations. H.S.T helped with analyzing the regional simulation. L.S., P.K., P.R., A.F.T., H.S.T. and M.F. reviewed the manuscript.

\section{Competing interests}

The authors declare no competing interests.

\section{Figure captions}

Fig. 1 Study region. Three-month seal's trajectory (black line) superimposed with a snapshot of chlorophyll a (colorscale) and SSH (white contours). Three regions are highlighted: the highly turbulent area (red), the weakly turbulent area (grey) and the southern eddy edge (orange). Cyclones (C) and anti-cyclones (A) are identified from elliptic SSH contours. Hyperbolic SSH contours located in-between eddies identify the strain field (see main text and Fig. 3a). Red arrows indicate the direction of the seal. Bathymetry contours of 0.5, 2 and $3 \mathrm{~km}$ (thin black lines) from NOAA ETOPO5 outline the Kerguelen plateau. The inset shows the Kerguelen region (red polygon). 
Fig. 2 Characteristics of the strongly turbulent area (in red in Fig. 1). a) Snapshot of SSH (colorbar) and geostrophic currents (black arrows) superimposed with surface buoyancy anomaly measured by the seal from 11/02/2014 to 11/24/2014. b) Along-seal-track time series of SSH (black line), seal-measured buoyancy anomaly (blue line) and steric height (green line, see Supplementary Information). c) Seal's vertical section of buoyancy. Thin black lines are isopycnals. d) Seal's vertical section of lateral buoyancy gradients, $b_{s}$. e) Seal's vertical section of the Richardson number. The mixed layer depth is shown in bold black on panels c)-e).

Fig. 3 Strain field, frontogenesis and vertical heat transport. a) Horizontal slice (x,y) of tracer patch (light blue) in a strain field (black arrows). The strain elongates (compresses) the tracer in the $y(x)$-direction ${ }^{10}$. Red (blue) Finite Size Lyapunov Exponents (FSLE, proxy of strain) identify horizontal stretching (compressing) directions. Fronts are aligned with stretching FSLE. b) 3-D slice of strain-induced submesoscale front. Thin black lines are isotherms. Vertical velocities (w, straight black arrows) develop in response to the front intensification. Since temperature and w anomalies are positively correlated, frontogenesis-induced vertical heat transport is upward (see Methods for a full description).

Fig. 4 FSLE and horizontal buoyancy gradients $\left(\left|b_{x}\right|\right)$. a) Snapshot of FSLE (colorscale) and SSH contours (dashed gray) for 11/13/2014 in the strongly turbulent region (red in Fig. 1), with surface $\left|b_{x}\right|$ from 11/02/2014 to 11/24/2014. b) Scatterplot of along-seal-track stretching FSLE and $\left|b_{x}\right|$ at $300 \mathrm{~m}$, over the entire trajectory, after a 30km-moving average and averaging the $\mathrm{y}$-axis over 100 bins (black dots), unaveraged data in gray. Relationship obtained between FSLE and $\left|b_{x}\right|$ in red. c) Along-seal-track time series of stretching FSLE (blue) and $\left|b_{x}\right|$ at $300 \mathrm{~m}$ (black) after a 5km-moving average, from km 1600 to 2100 .

Fig. 5 Temperature anomaly, vertical velocity and vertical heat transport (VHT). Vertical section of a) seal-sampled temperature anomaly, b) vertical velocity derived from seal and satellite data with equation 3, c) VHT, as defined in the Methods. In a)-c), the mixed layer depth is in bold black. RMS(z) from km 1000 to 2000 of d) temperature anomaly, e) vertical velocity. f) averaged VHT $(<\mathrm{VHT}\rangle)$. $<\mathrm{VHT}>$ reaches $\sim+110 \mathrm{~W} / \mathrm{m}^{2}$ at $200 \mathrm{~m}$. The percentage of profiles (in gray) goes from $100 \%$ at $200 \mathrm{~m}$ to $40 \%$ at $400 \mathrm{~m}$.

Extended Data Fig. 1 Weakly turbulent and southern eddy edge areas Same as Fig. 2 but for i) 2014/11/24 to 2014/12/20 with the SSH snapshot in a) taken on 2014/12/07. The seal crosses a large anti-cyclonic region (grey trajectory in Fig. 1) characterized by weaker currents (smaller SSH anomalies) and referred to as the weakly turbulent area. ii) 2014/12/22-29 with the SSH snapshot in a) taken on 2014/12/26. The seal follows the edges of mesoscale eddies over a distance of $\sim 600 \mathrm{~km}$. This region is referred to as the southern eddy (in orange in Fig. 1). Bold black arrows indicate the direction of the seal.

Extended Data Fig. 2 Lateral gradient of buoyancy and Richardson number in the strongly turbulent area. a) RMS of lateral gradients of buoyancy, $\left|b_{x}\right|$, as a function of depth in the strongly turbulent area. b) Scatter plot between lateral gradients of buoyancy, $\left|b_{x}\right|$, and Richardson number, $R i$, in the strongly turbulent area. $R i<2$ coincide with strong buoyancy gradients $\left(\left|b_{x}\right|>2.5 \times 10^{-7} \mathrm{~s}^{-2}\right)$, highlighting the ageostrophic character of the dynamical regime encountered by the seal and the expected strong frontogenesis processes at play.

Extended Data Fig. 3 Map of finite size Lyapunov exponents Map of FSLE over the entire domain on 13 November 2014. FSLE are greatly enhanced in the strongly turbulent region (black rectangle and in red in Fig. 1) compared to the rest of the domain.

Extended Data Fig. 4 Finite size Lyapunov exponents and horizontal gradient of buoyancy, vertical velocities and vertical heat transport at $\mathbf{3 0 0} \mathbf{~ m}$. Times series of a) Horizontal gradients of buoyancy at $300 \mathrm{~m}$ sampled by the seal (in black) and FSLE derived from satellite altimetry along the seal's track (in blue). b) Vertical velocities at $300 \mathrm{~m}$ derived from the seal and satellite data by solving the omega equation (see main text and Methods). c) Vertical heat transport (see Methods). The areas described in the main text and in Fig. 1 are highlighted by the colored rectangles. 
Extended Data Fig. 5 Daily averaged vertical velocities and vertical heat transport from the high-resolution numerical simulation. Daily averaged vertical section from the high-resolution numerical simulation for November 22,2011 at $52^{\circ} \mathrm{S}$ of a) Vertical velocities b) Vertical heat transport. Isopycnals are shown by the black lines. Enhanced vertical velocities and heat transport with a width of 5-10 km are found in the ocean interior and, in particular, below the mixed layer, similar to the observation presented in the main text.

Extended Data Fig. 6 Averaged vertical heat transport from the high-resolution numerical simulation. 2-D (x,y) view of 10-day averaged vertical heat transport (VHT) at a) $50 \mathrm{~m}$ and b) $200 \mathrm{~m}$. Isotherms are shown in black. Domain averaged values are respectively 92 and $197 \mathrm{~W} / \mathrm{m}^{2}$. VHT is enhanced at depth and follows the isotherms on the eddy edges, and its averaged value is directed upward (positive value), all of which is consistent with the observational results presented in the main text.

Extended Data Fig. 7 Domain averaged vertical heat transport from the high-resolution numerical simulation. Monthly averaged vertical heat transport $(<\mathrm{VHT}\rangle)$ ) as a function of depth over the entire domain from the high-resolution numerical simulation. VHT is directed upwards (positive values) and its magnitude is similar - although even higher - than what is derived from the observational data presented in the main text.

Extended Data Fig. 8 Distance between two dives and angle between the seal's trajectory and the fronts. a) Histogram of the distance between two dives. Median distance between two dives is $700 \mathrm{~m}$ (dotted line) and the shortest distance is $100 \mathrm{~m}$. b) Histogram of the angle between the seal's trajectory and the stretching FSLE it encounters for FSLE $>0.15$ day $^{-1}$. Oblique crossings are most frequent and a normalization is implemented to correct for it (see Methods). 


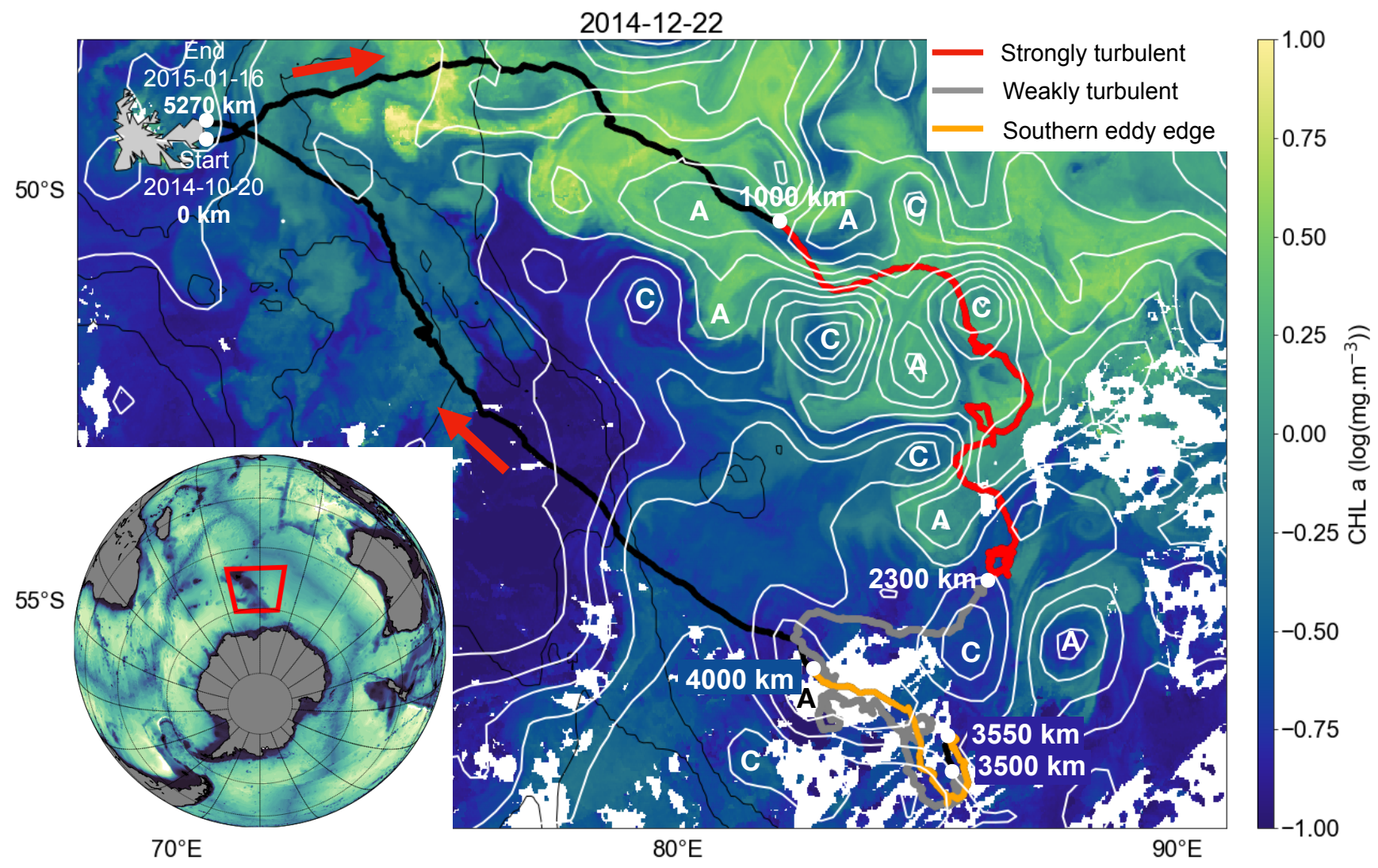

Figure 1. Study region. Three-month seal's trajectory (black line) superimposed with a snapshot of chlorophyll a (colorscale) and SSH (white contours). Three regions are highlighted: the highly turbulent area (red), the weakly turbulent area (grey) and the southern eddy edge (orange). Cyclones (C) and anti-cyclones (A) are identified from elliptic SSH contours. Hyperbolic SSH contours located in-between eddies identify the strain field (see main text and Fig. 3a). Red arrows indicate the direction of the seal. Bathymetry contours of 0.5, 2 and $3 \mathrm{~km}$ (thin black lines) from NOAA ETOPO5 outline the Kerguelen plateau. The inset shows the Kerguelen region (red polygon). 


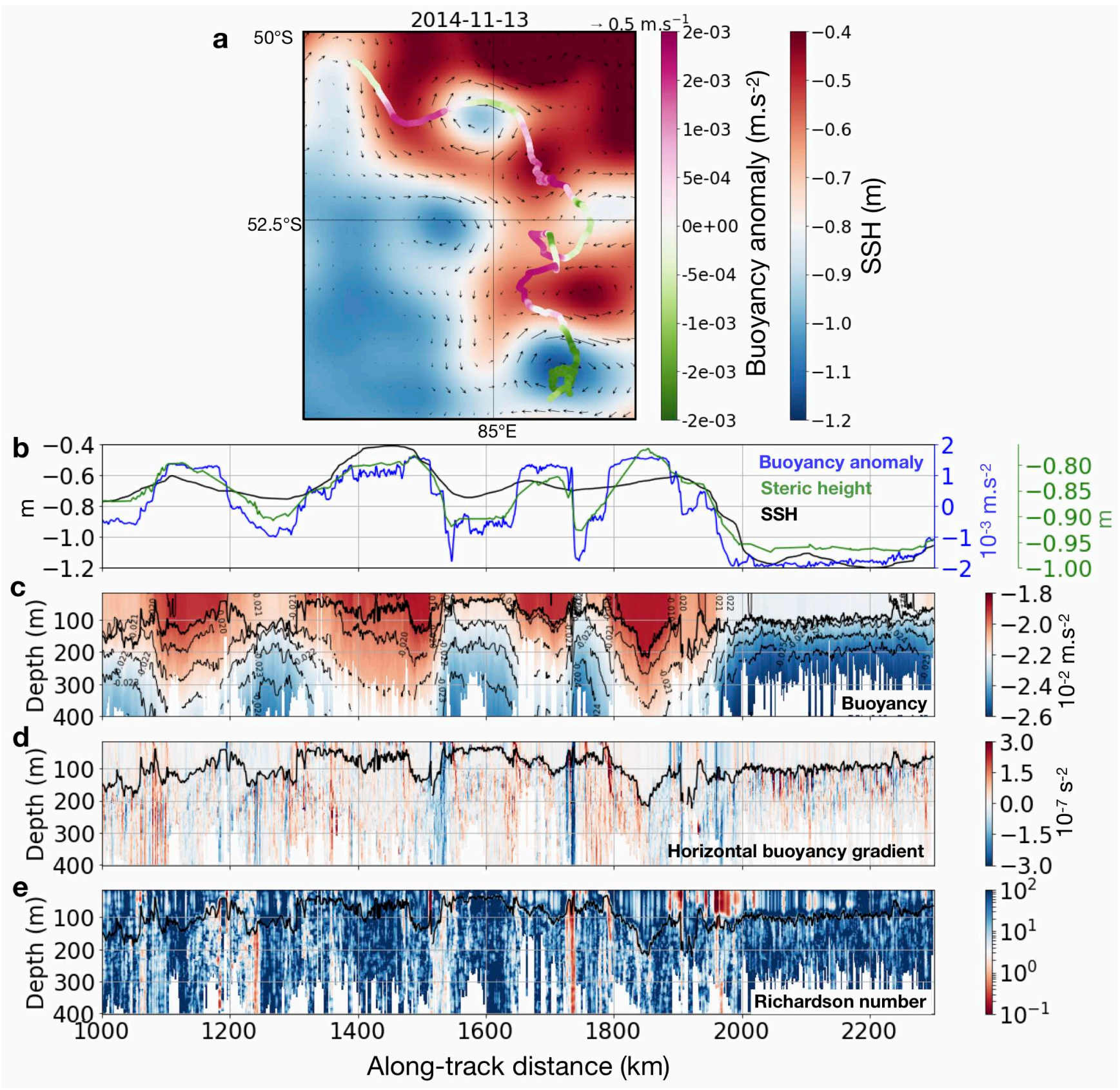

Figure 2. Characteristics of the strongly turbulent area (in red in Fig. 1). a) Snapshot of SSH (colorbar) and geostrophic currents (black arrows) superimposed with surface buoyancy anomaly measured by the seal from 11/02/2014 to 11/24/2014. b) Along-seal-track time series of SSH (black line), seal-measured buoyancy anomaly (blue line) and steric height (green line, see Supplementary Information). c) Seal's vertical section of buoyancy. Thin black lines are isopycnals. d) Seal's vertical section of lateral buoyancy gradients, $b_{s}$. e) Seal's vertical section of the Richardson number. The mixed layer depth is shown in bold black on panels c)-e). 
a

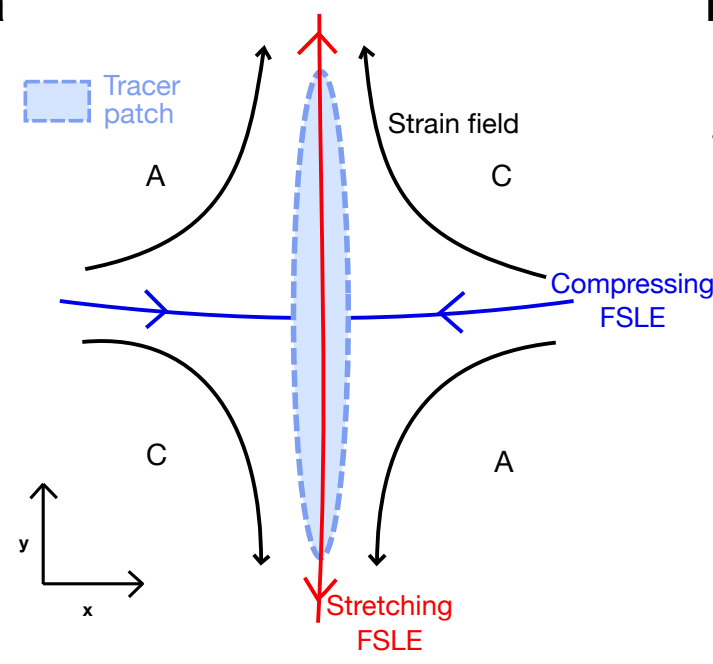

b

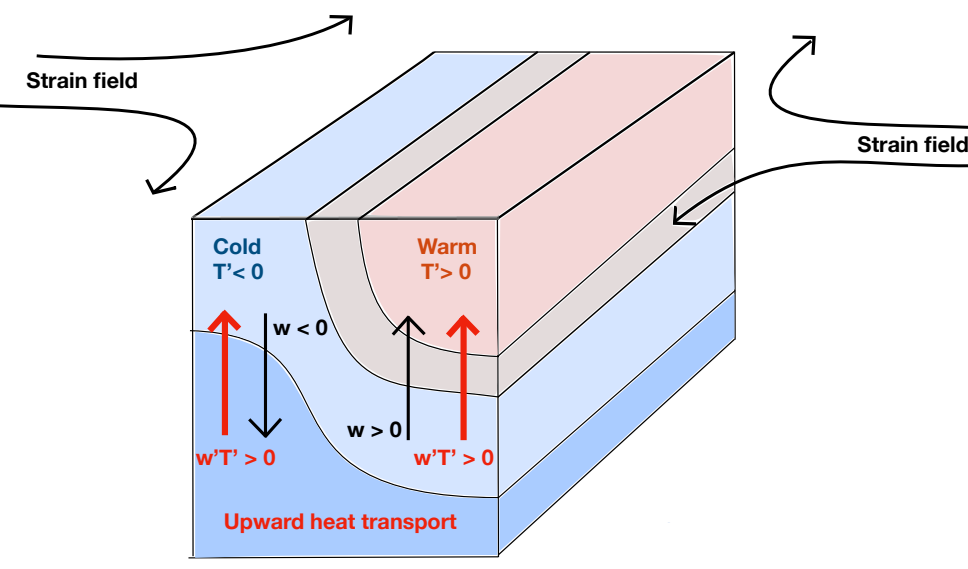

Figure 3. Strain field, frontogenesis and vertical heat transport. a) Horizontal slice (x,y) of tracer patch (light blue) in a strain field (black arrows). The strain elongates (compresses) the tracer in the $\mathrm{y}(\mathrm{x})$-direction ${ }^{10}$. Red (blue) Finite Size Lyapunov Exponents (FSLE, proxy of strain) identify horizontal stretching (compressing) directions. Fronts are aligned with stretching FSLE. b) 3-D slice of strain-induced submesoscale front. Thin black lines are isotherms. Vertical velocities (w, straight black arrows) develop in response to the front intensification. Since temperature and $\mathrm{w}$ anomalies are positively correlated, frontogenesis-induced vertical heat transport is upward (see Methods for a full description). 

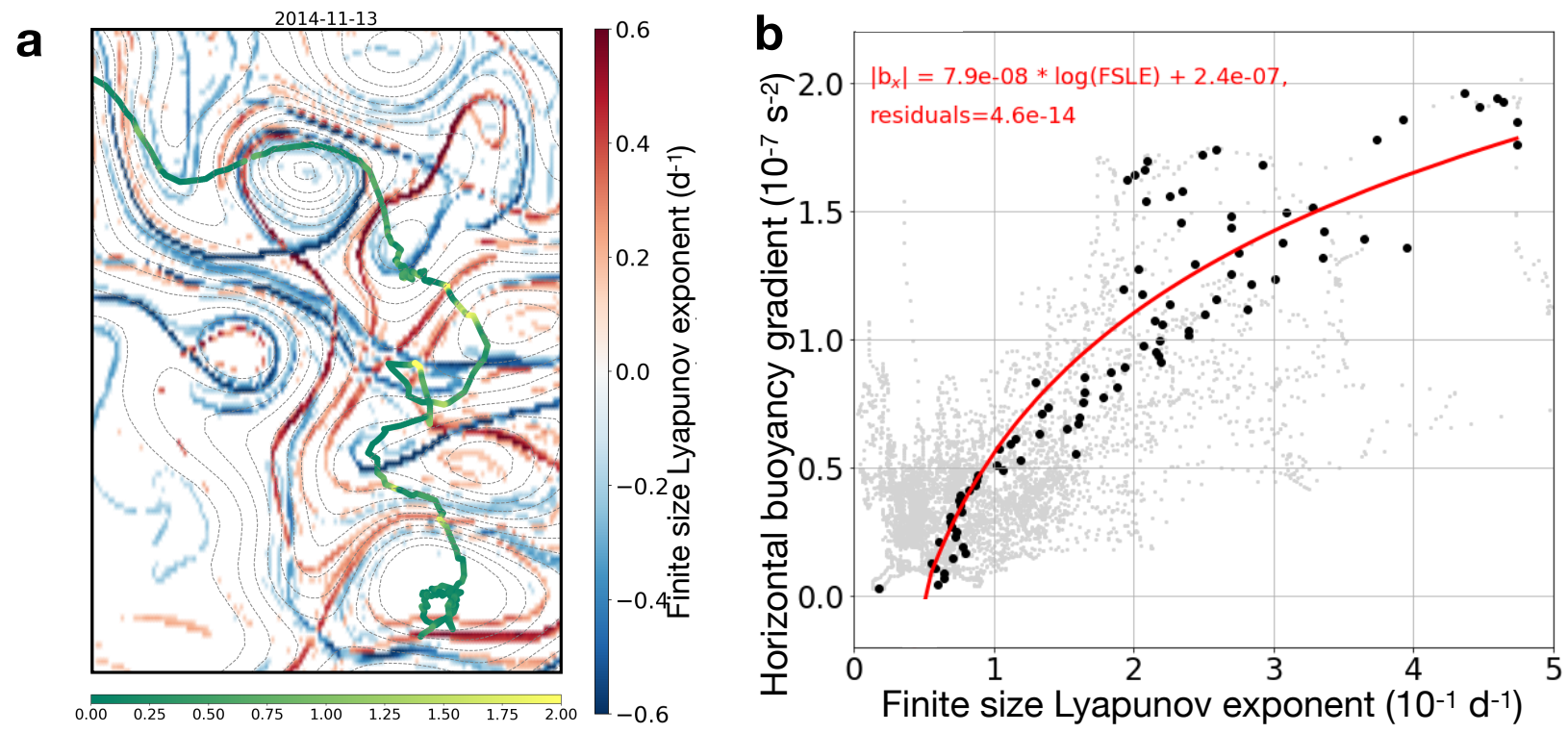

Horizontal buoyancy gradient $\left(10^{-7} \mathrm{~s}^{-2}\right)$

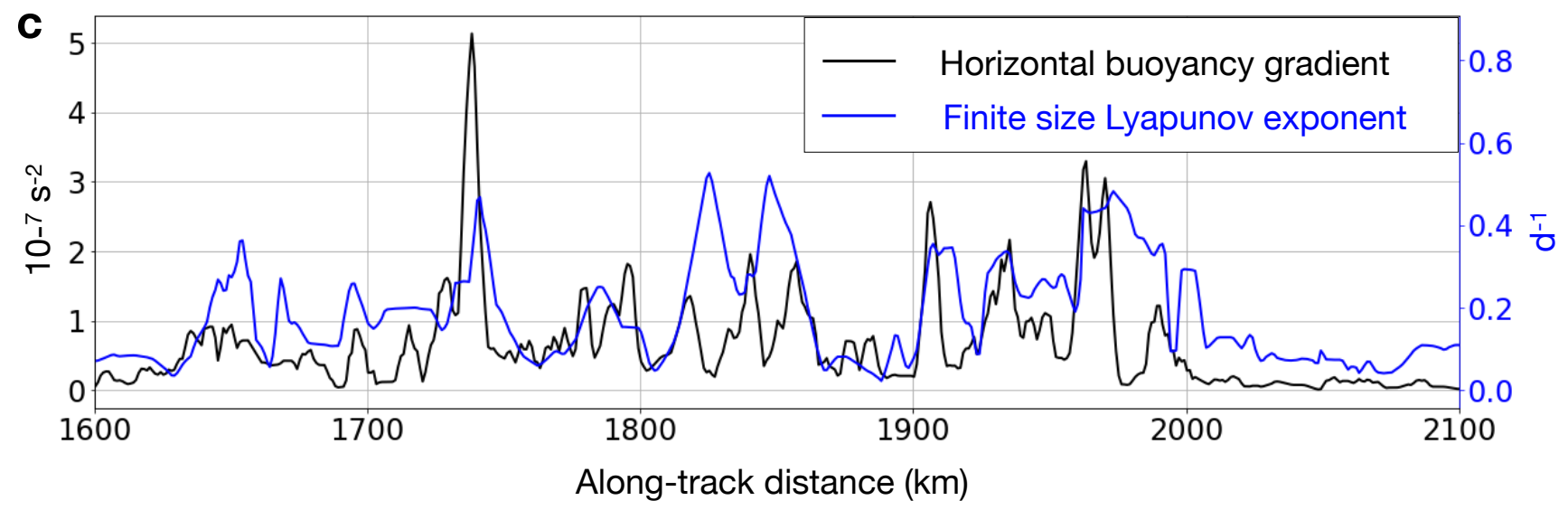

Figure 4. FSLE and horizontal buoyancy gradients $\left(\left|b_{x}\right|\right)$. a) Snapshot of FSLE (colorscale) and SSH contours (dashed gray) for 11/13/2014 in the strongly turbulent region (red in Fig. 1), with surface $\left|b_{x}\right|$ from 11/02/2014 to 11/24/2014. b) Scatterplot of along-seal-track stretching FSLE and $\left|b_{x}\right|$ at $300 \mathrm{~m}$, over the entire trajectory, after a 30km-moving average and averaging the y-axis over 100 bins (black dots), unaveraged data in gray. Relationship obtained between FSLE and $\left|b_{x}\right|$ in red. c) Along-seal-track time series of stretching FSLE (blue) and $\left|b_{x}\right|$ at $300 \mathrm{~m}$ (black) after a 5km-moving average, from km 1600 to 2100 . 

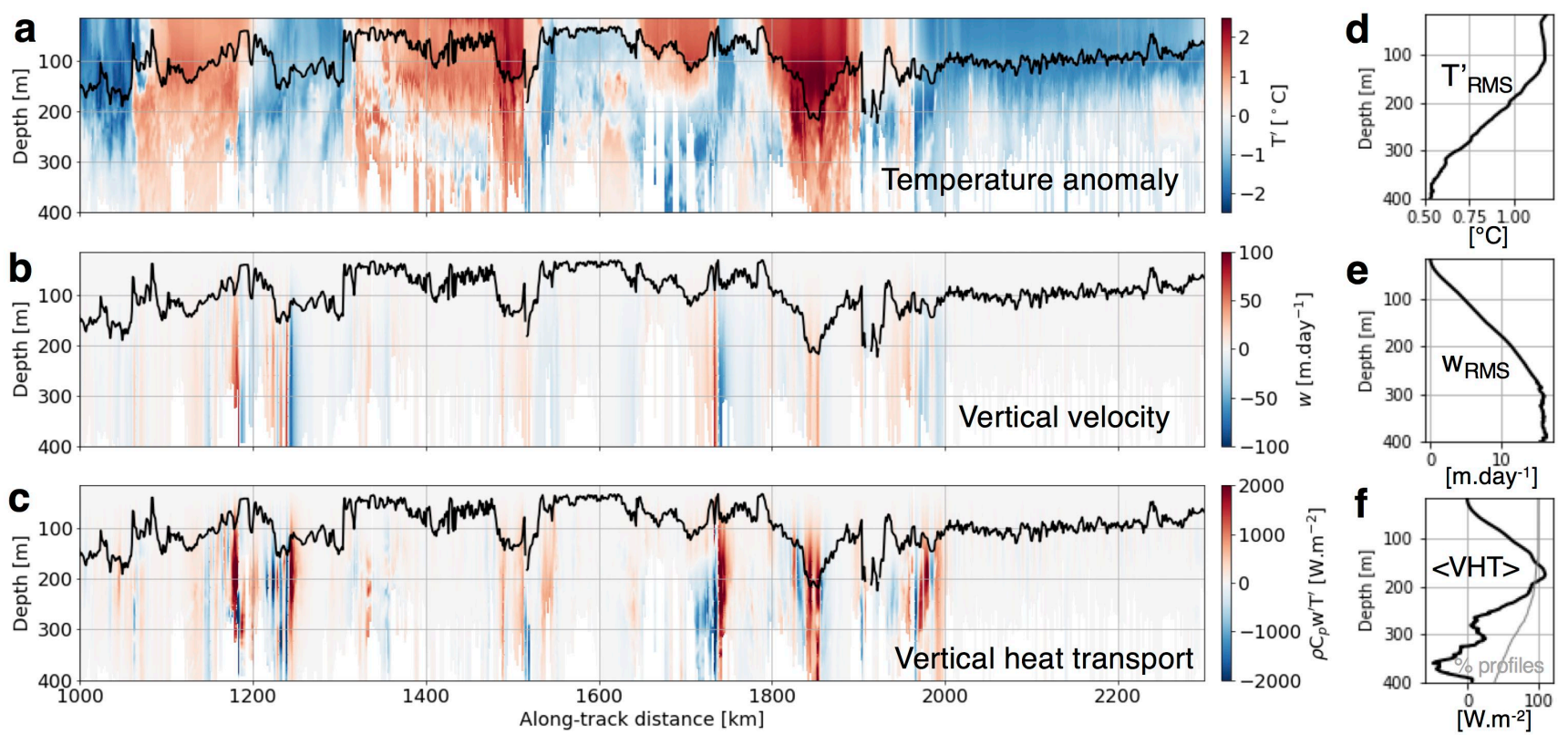

Figure 5. Temperature anomaly, vertical velocity and vertical heat transport (VHT). Vertical section of a) seal-sampled temperature anomaly, b) vertical velocity derived from seal and satellite data with equation 3, c) VHT, as defined in the Methods. In a)-c), the mixed layer depth is in bold black. RMS(z) from km 1000 to 2000 of d) temperature anomaly, e) vertical velocity. f) averaged VHT $(<\mathrm{VHT}>)$. $<\mathrm{VHT}>$ reaches $\sim+110 \mathrm{~W} / \mathrm{m}^{2}$ at $200 \mathrm{~m}$. The percentage of profiles (in gray) goes from $100 \%$ at $200 \mathrm{~m}$ to $40 \%$ at $400 \mathrm{~m}$. 

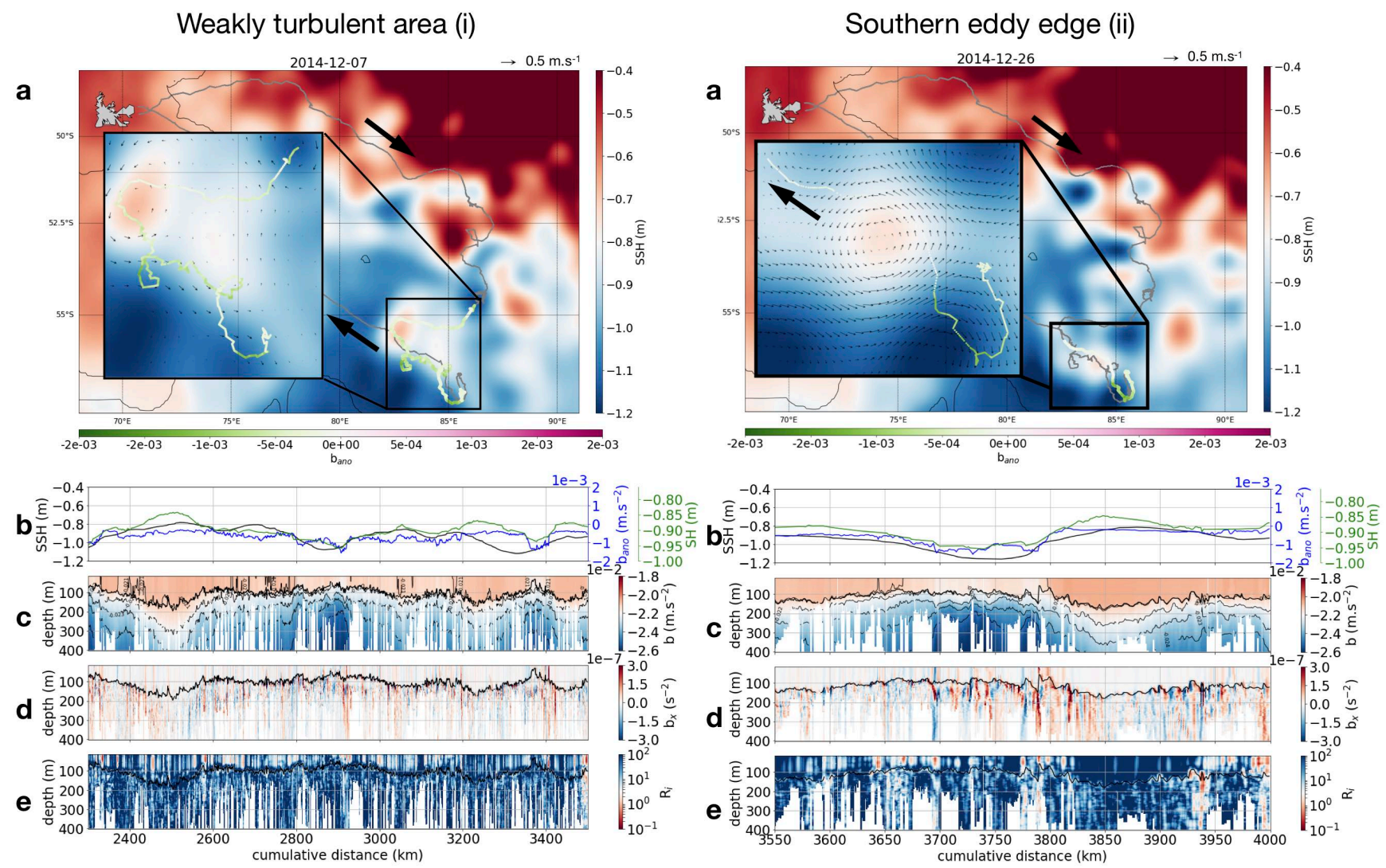

Extended Data Fig. 1. Weakly turbulent and southern eddy edge areas Same as Fig. 2 but for i) 2014/11/24 to 2014/12/20 with the SSH snapshot in a) taken on 2014/12/07. The seal crosses a large anti-cyclonic region (grey trajectory in Fig. 1) characterized by weaker currents (smaller SSH anomalies) and referred to as the weakly turbulent area. ii) 2014/12/22-29 with the SSH snapshot in a) taken on 2014/12/26. The seal follows the edges of mesoscale eddies over a distance of $\sim 600 \mathrm{~km}$. This region is referred to as the southern eddy (in orange in Fig. 1). Bold black arrows indicate the direction of the seal. 

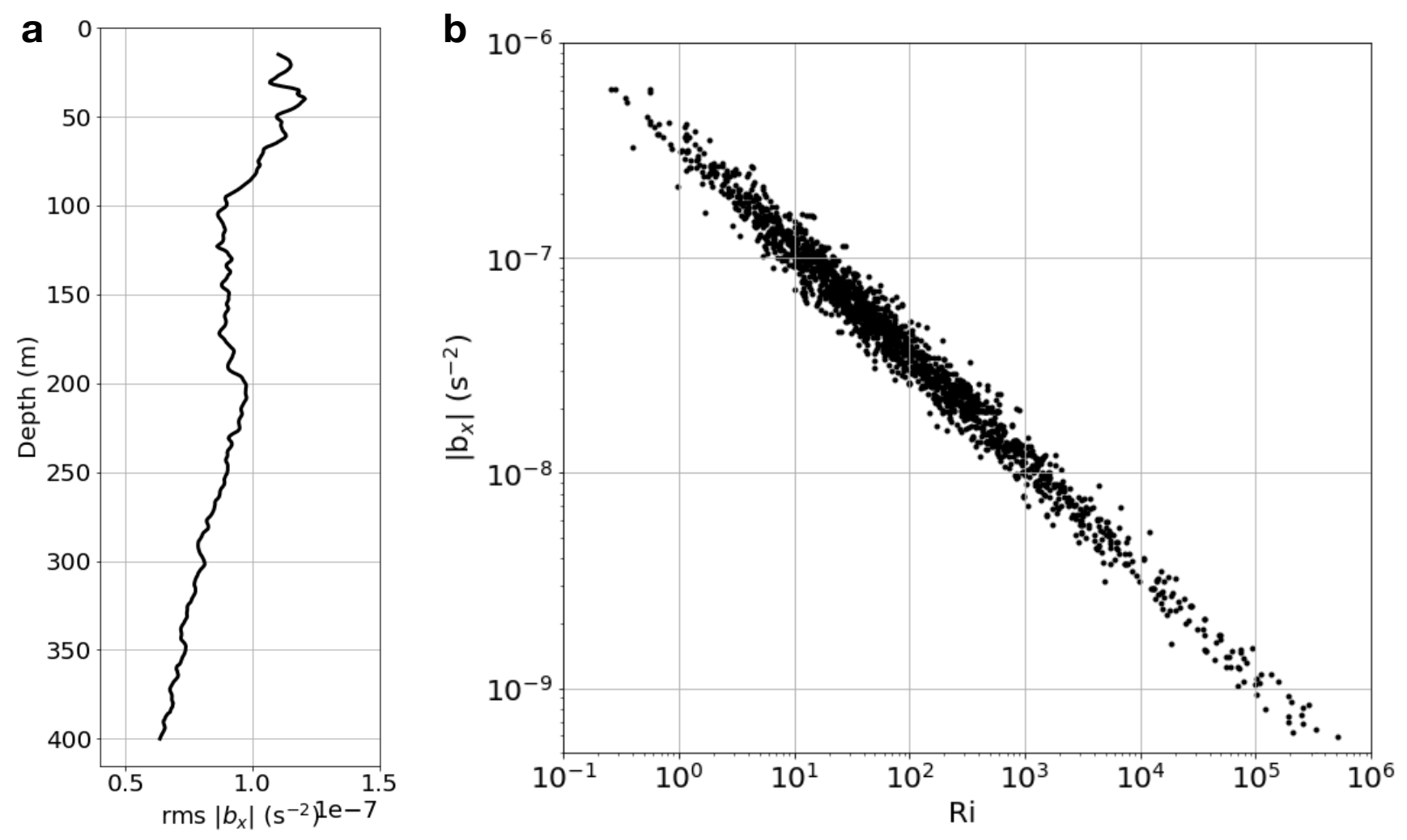

Extended Data Fig. 2. Lateral gradient of buoyancy and Richardson number in the strongly turbulent area. a) RMS of lateral gradients of buoyancy, $\left|b_{x}\right|$, as a function of depth in the strongly turbulent area. b) Scatter plot between lateral gradients of buoyancy, $\left|b_{x}\right|$, and Richardson number, $R i$, in the strongly turbulent area. $R i<2$ coincide with strong buoyancy gradients $\left(\left|b_{x}\right|>2.5 \times 10^{-7} \mathrm{~s}^{-2}\right)$, highlighting the ageostrophic character of the dynamical regime encountered by the seal and the expected strong frontogenesis processes at play. 


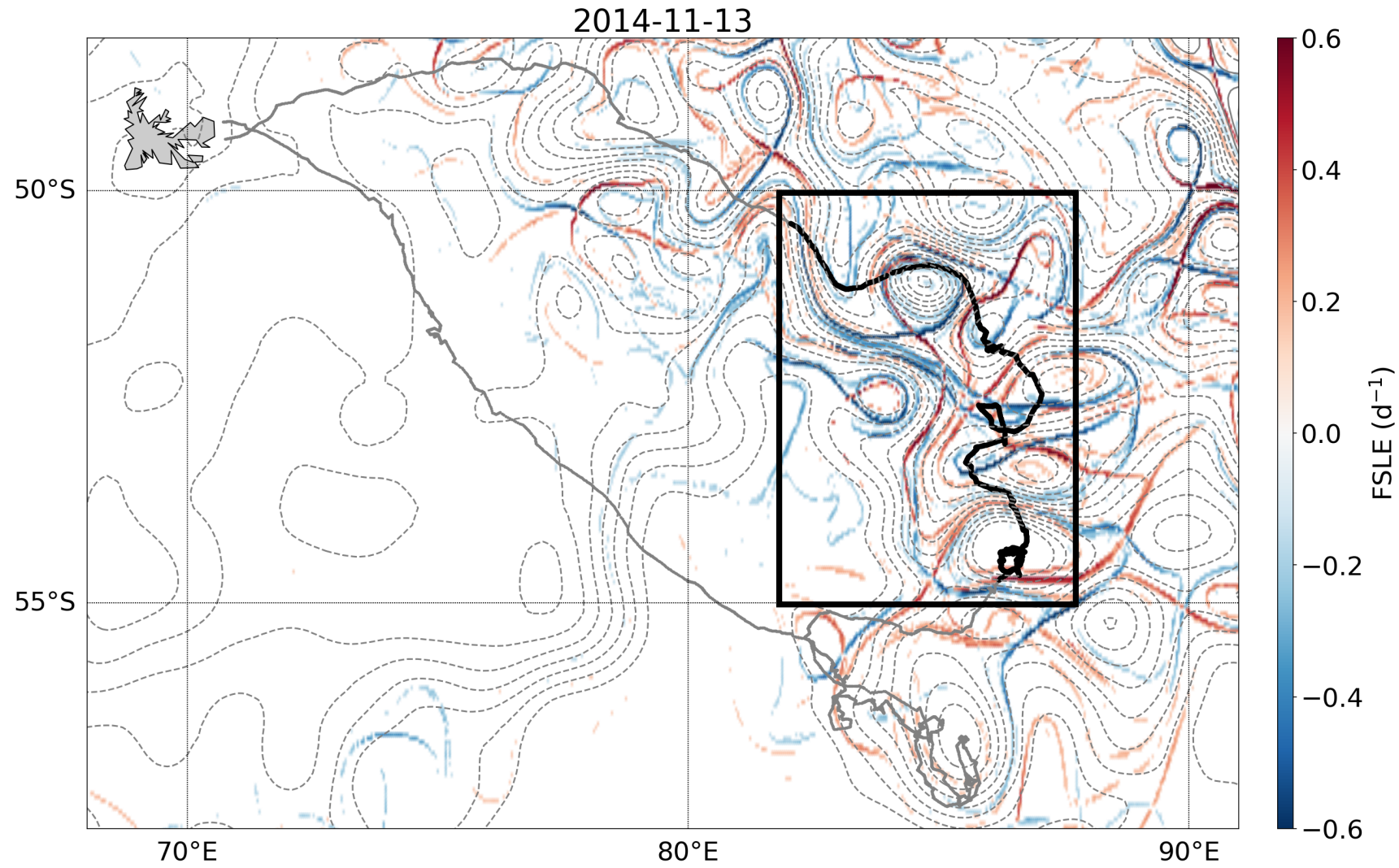

Extended Data Fig. 3. Map of finite size Lyapunov exponents Map of FSLE over the entire domain on 13 November 2014. FSLE are greatly enhanced in the strongly turbulent region (black rectangle and in red in Fig. 1) compared to the rest of the domain. 


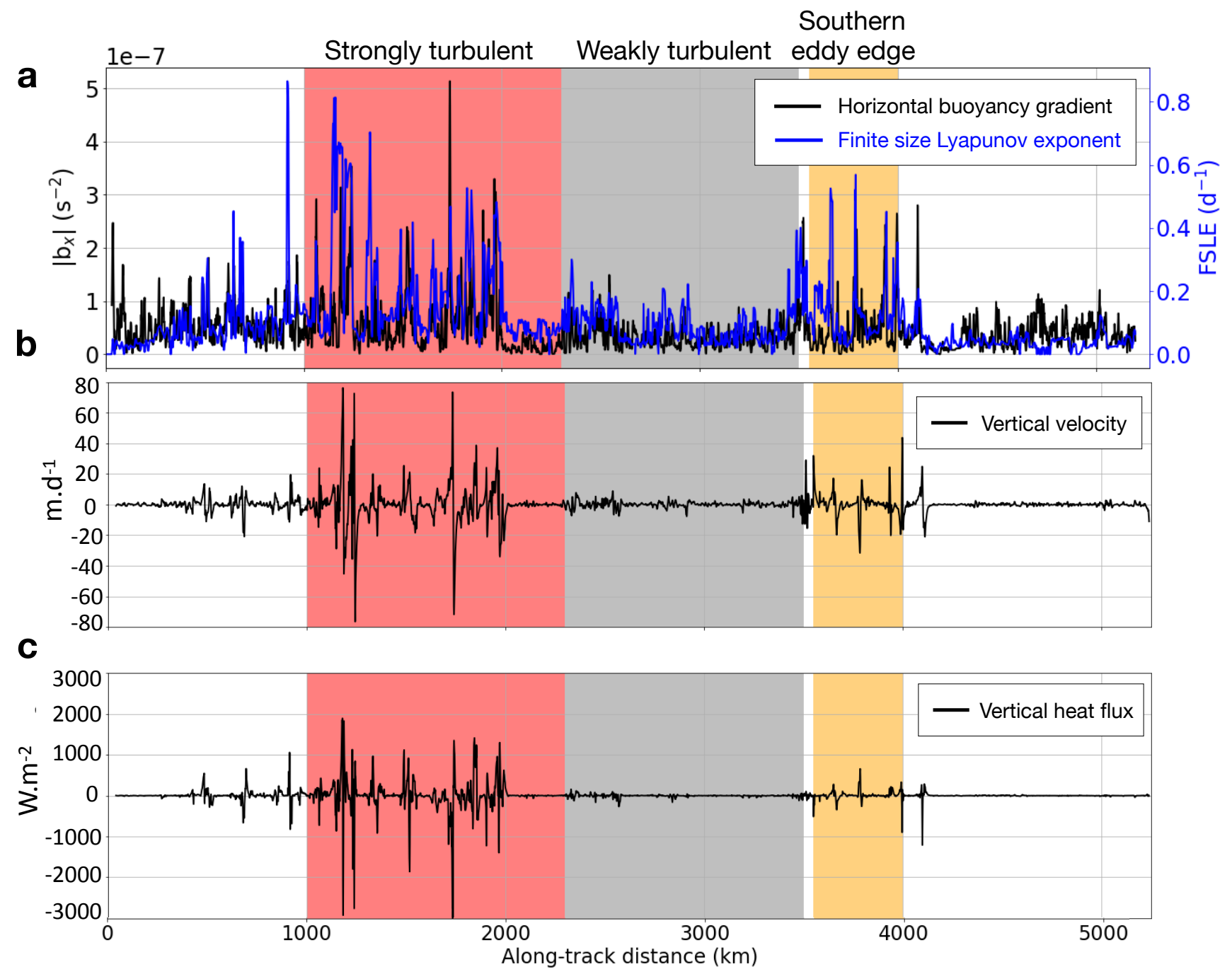

Extended Data Fig. 4. Finite size Lyapunov exponents and horizontal gradient of buoyancy, vertical velocities and vertical heat transport at $300 \mathrm{~m}$. Times series of a) Horizontal gradients of buoyancy at $300 \mathrm{~m}$ sampled by the seal (in black) and FSLE derived from satellite altimetry along the seal's track (in blue). b) Vertical velocities at $300 \mathrm{~m}$ derived from the seal and satellite data by solving the omega equation (see main text and Methods). c) Vertical heat transport (see Methods). The areas described in the main text and in Fig. 1 are highlighted by the colored rectangles. 


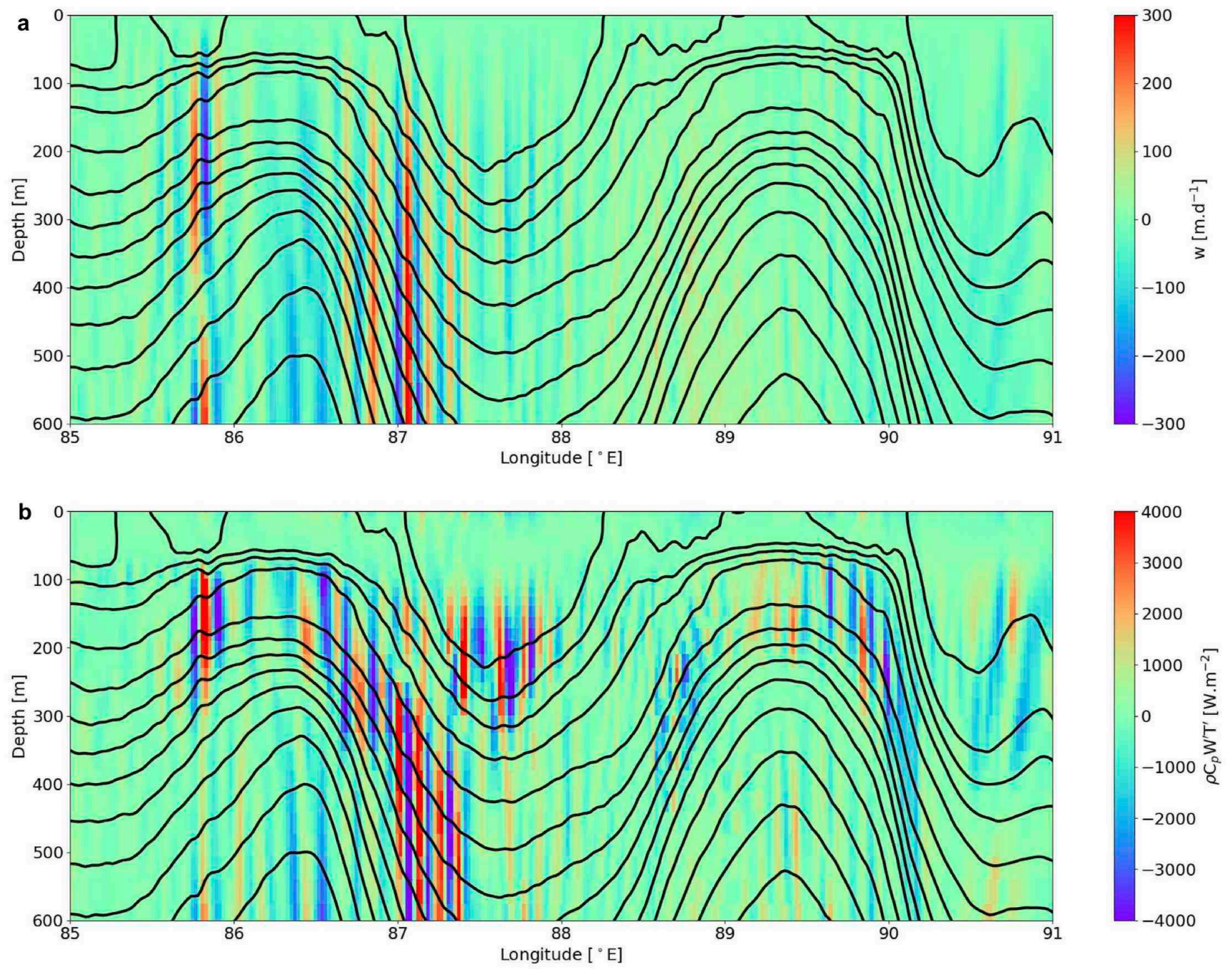

Extended Data Fig. 5. Daily averaged vertical velocities and vertical heat transport from the high-resolution

numerical simulation. Daily averaged vertical section from the high-resolution numerical simulation for November 22, 2011 at $52^{\circ} \mathrm{S}$ of a) Vertical velocities b) Vertical heat transport. Isopycnals are shown by the black lines. Enhanced vertical velocities and heat transport with a width of 5-10 km are found in the ocean interior and, in particular, below the mixed layer, similar to the observation presented in the main text. 


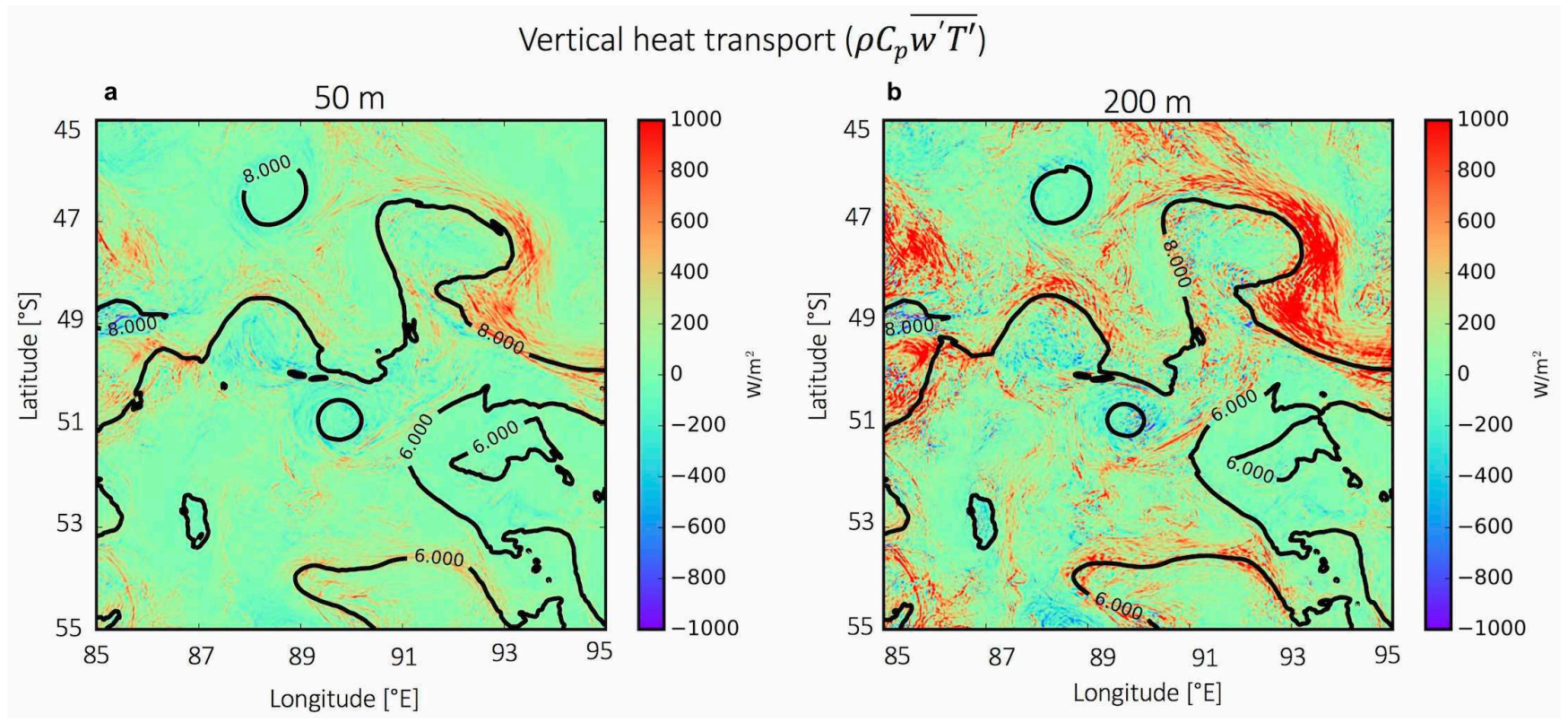

Extended Data Fig. 6. Averaged vertical heat transport from the high-resolution numerical simulation. 2-D (x,y) view of 10-day averaged vertical heat transport (VHT) at a) $50 \mathrm{~m}$ and b) $200 \mathrm{~m}$. Isotherms are shown in black. Domain averaged values are respectively 92 and $197 \mathrm{~W} / \mathrm{m}^{2}$. VHT is enhanced at depth and follows the isotherms on the eddy edges, and its averaged value is directed upward (positive value), all of which is consistent with the observational results presented in the main text. 


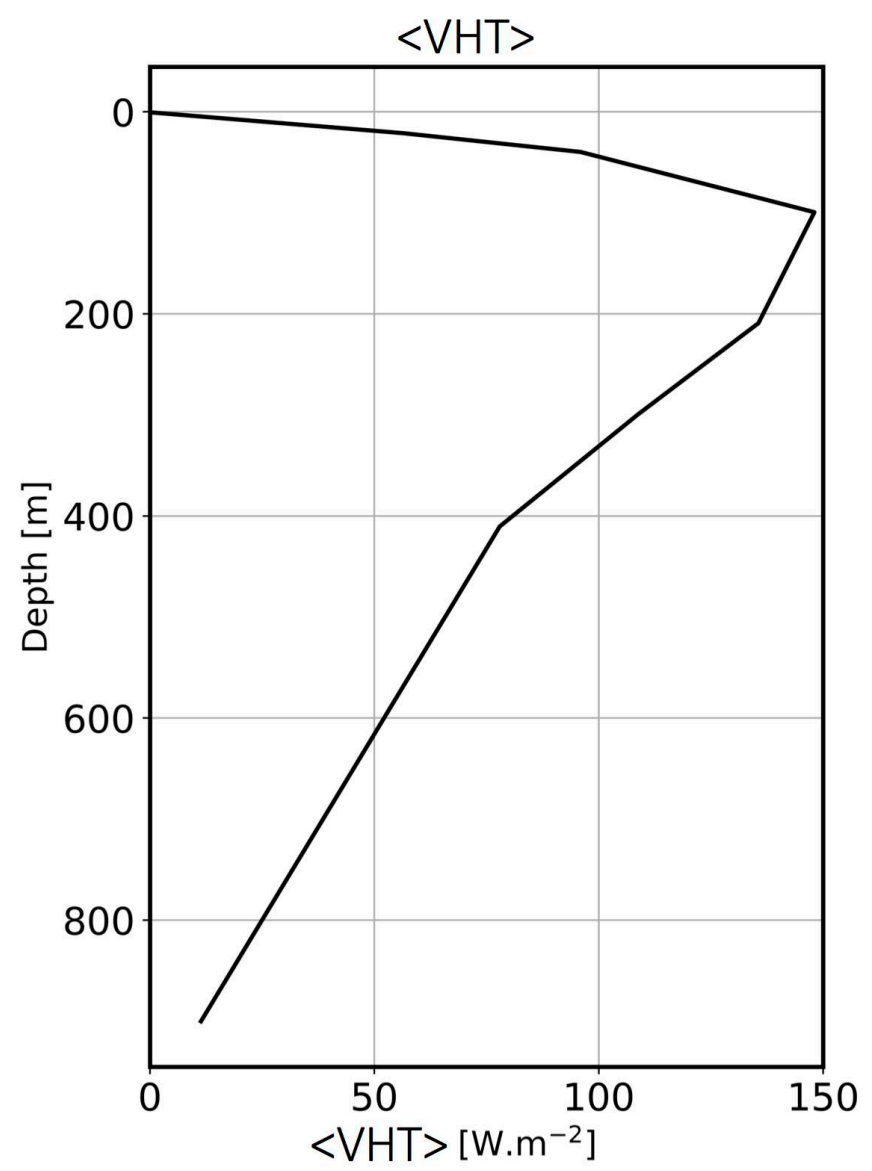

Extended Data Fig. 7. Domain averaged vertical heat transport from the high-resolution numerical simulation. Monthly averaged vertical heat transport $(<\mathrm{VHT}\rangle)$ as a function of depth over the entire domain from the high-resolution numerical simulation. VHT is directed upwards (positive values) and its magnitude is similar - although even higher - than what is derived from the observational data presented in the main text.
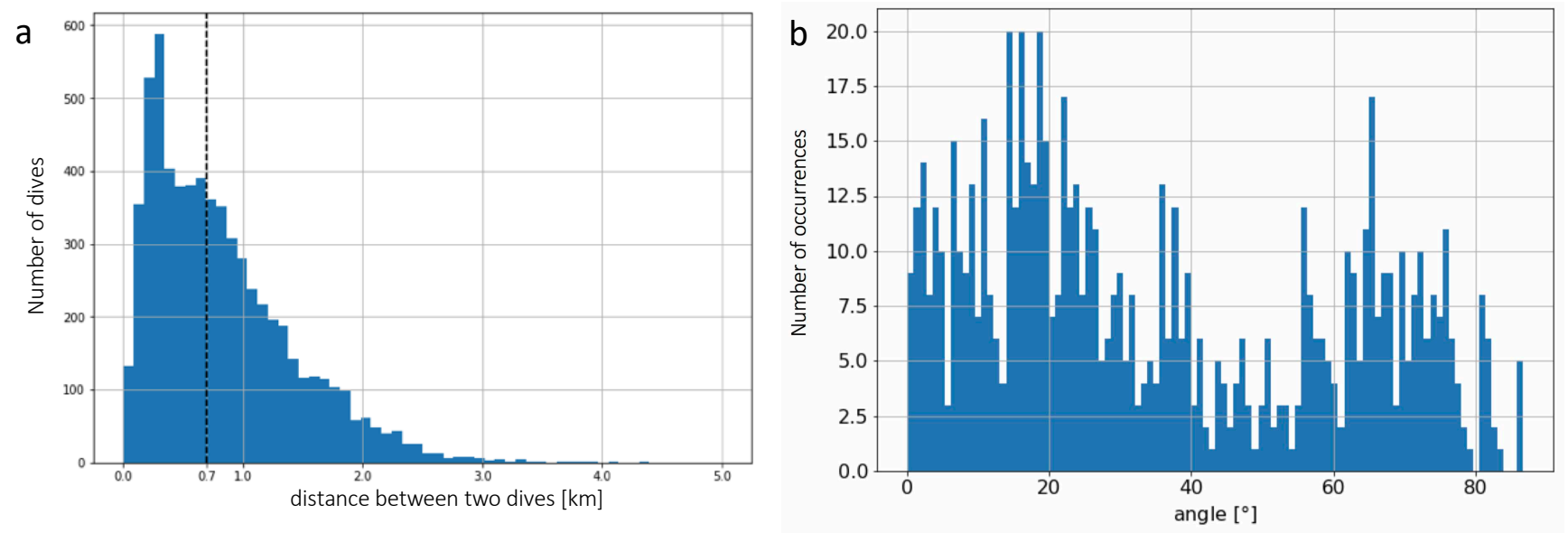

Extended Data Fig. 8. Distance between two dives and angle between the seal's trajectory and the fronts. a) Histogram of the distance between two dives. Median distance between two dives is $700 \mathrm{~m}$ (dotted line) and the shortest distance is $100 \mathrm{~m}$. b) Histogram of the angle between the seal's trajectory and the stretching FSLE it encounters for FSLE $>0.15$ day $^{-1}$. Oblique crossings are most frequent and a normalization is implemented to correct for it (see Methods). 


\title{
Enhanced upward heat transport at deep submesoscale ocean fronts - Supplementary Information
}

\author{
Lia Siegelman ${ }^{1,2,3^{*}}$, Patrice Klein ${ }^{1,2,4}$, Pascal Rivière ${ }^{3}$, Andrew F. Thompson ${ }^{1}$, Hector S. \\ Torres $^{2}$, Mar Flexas ${ }^{1}$, and Dimitris Menemenlis ${ }^{2}$
}

${ }^{1}$ Environmental Science and Engineering, California Institute of Technology, Pasadena, CA, USA

2Jet Propulsion Laboratory, California Institute of Technology, Pasadena, CA, USA

${ }^{3}$ Univ. Brest, CNRS, IRD, Ifremer, LEMAR, Plouzané, France

${ }^{4}$ Univ. Brest, CNRS, IRD, Ifremer, LOPS, Plouzané, France

*Isiegelman@caltech.edu

\section{Consistency between seal and satellite observations at meso- and submesoscale}

The contribution of the vertical stratification to the SSH can be estimated by integrating the hydrostatic equation $\mathrm{d} p / \mathrm{d} z=-\rho g$. The $\operatorname{SSH}(\eta)$ referenced from $z=0$ is then given by ${ }^{1}$

$$
\eta=\frac{p_{b}^{\prime}}{\rho_{0} g}-\frac{p_{a}}{\rho_{0} g}-\int_{-H}^{0} \frac{\rho^{\prime}}{\rho_{0}} d z
$$

where $p$ is the pressure, $\rho$ the potential density, $\rho_{0}$ the reference potential density $\left(1025 \mathrm{~kg} \mathrm{~m}^{-3}\right), \rho^{\prime}$ the potential density anomaly $\left(\rho=\rho_{0}+\rho^{\prime}\right), g$ the gravity of Earth, $p_{a}$ the atmospheric surface pressure, $H$ the depth of the ocean and $p_{b}^{\prime}=p_{b}-\rho_{0} g H$ the bottom pressure anomaly with $p_{b}$ the ocean bottom pressure. The term $\int_{0}^{\eta} \rho^{\prime} / \rho_{0} d z$ has been neglected because $\eta \ll H$. The three terms on the right hand side of equation (1) represent respectively the contributions from the bottom pressure, the atmospheric pressure loading, and the steric height. The steric height $(\zeta)$ is computed with the seal dataset from the surface down to $H=400 \mathrm{~m}$ for dives reaching at least $400 \mathrm{~m}$ (3100 dives) as $\zeta_{400}=\int_{-400}^{0} \rho^{\prime} / \rho_{0} d z$. $\zeta_{400}$ is then compared to the SSH observed by altimetry.

SSH obtained from satellite data and mixed layer depth (MLD) and buoyancy anomalies, sampled by the seal, have a larger magnitude in the strongly turbulent region (Fig. 2) than in the weakly turbulent one (Extended Data Fig. 1i). Anticyclonic structures, or positive SSH anomalies, are associated with an increase in MLD and bowl-shaped positive buoyancy anomalies from the surface down to $400 \mathrm{~m}$ depth (Fig. 2 and Extended Data Fig. 1). Shallow MLD and negative buoyancy anomalies are associated with cyclonic structures, or negative SSH anomalies, confirming the fact that buoyancy anomalies compensate SSH anomalies. To further quantify this compensation over the water column, we have compared SSH with the depth-integrated buoyancy also called steric height. Since seal observations mostly sample the upper ocean, the steric height contribution has been estimated from surface down to $400 \mathrm{~m}$ (which concerns at least $35 \%$ of the dives) as $\zeta_{400}=\int_{-400}^{0} b d z$ with $b$ the buoyancy (see Methods). As emphasized by the time-series in Fig. S1a (SSH in black and $\zeta_{400}$ in blue), SSH and steric height fluctuations correlate well over the entire trajectory, but only for scales smaller than $100 \mathrm{~km}$. SSH reveals a large-scale signal $(\sim 1000 \mathrm{~km})$ not present for $\zeta_{400}$. This indicates that SSH at larger scales is likely compensated by the buoyancy field below $400 \mathrm{~m}$ and/or by bottom pressure, The contribution of $\zeta_{400}$ to SSH is no more than $30 \%$ as revealed by the linear regression coefficient of 3.58 linking the two-time series (not shown).

To remove this large-scale contribution and focus only on the meso/sub-mesoscale band, time-series of the horizontal gradients of SSH and $\zeta_{400}$ are computed (a gradient operator overemphasizes contribution of smaller scales). This is the equivalent of comparing horizontal currents from SSH using the geostrophic approximation, with those explained by the buoyancy field contribution in the water column using the thermal wind balance ${ }^{1}$. The two time-series are now closer in terms of amplitude differences (Fig. S1b). $\zeta_{400}$ gradients statistically explain almost $75 \%$ of the SSH gradients as revealed by the linear regression coefficient of 1.35 linking the two time-series (not shown). This further emphasizes the consistency between the two independent datasets at meso/sub-mesoscale despite their different spatial resolution. The main differences concern the extrema of SSH gradients (mostly located on the eddy edges) that overcome those of $\zeta_{400}$ gradients by a factor of two to three. This suggests the steric height is not integrated deep enough to capture SSH in the upper mesoscale band and therefore that dynamics of this band affects at least the first $400 \mathrm{~m}$ below the surface. 


\section{Link between the strain field, Finite Size Lyapunov Exponents and lateral gradients of buoyancy}

As illustrated in Fig. 3a, buoyancy anomalies embedded in a strain field are elongated in one direction and compressed in the perpendicular direction. This mechanism can be understood in terms of particle dispersion, and thus in terms of Finite Size Lyapunov Exponents (FSLE); two particles, initially close and embedded in a strain field, become quickly separated in one particular direction (the stretching direction, in red in Figs 3a and 4a). As such, the time scale of their separation can be characterized by $\lambda^{-1}$ (see equation 2 in Methods), with large $\lambda$ being indicative of an intense strain field. More precisely, FSLE characterize both the time scale (via the largest FLSE eigenvalue $\lambda$ ) and the direction (via the FSLE eigenvector associated to $\lambda$ ) of the elongation of buoyancy anomalies.

Thus, the time scale $\lambda$ (shown in the colorbar of Fig. 4a for instance) can be used to characterize the production of buoyancy gradient expressed in the RHS of the omega equation (equation 3 in Methods). In particular, the intersection of intense compressing and stretching FSLE lines, respectively in blue and red in Figs 3a and 4a, identify Lagrangian hyperbolic points, where particles (or tracers) are simultaneously being stretched along one direction and compressed along the other one. Areas surrounding hyperbolic points are particularly prone to frontogenesis.

\section{Vertical velocities underestimation}

At steeply titled isopycnals' fronts, the vertical velocity field is more accurately captured by the full omega equation, known as the Sawyer-Eliassen (SE) equation, than by its QG version ${ }^{2,3}$. The 2-D (x,z) SE equation, assuming the front is embedded in a pure strain field and diffusive processes are negligible, reads ${ }^{2}$ :

$$
N^{2} \frac{\partial^{2} \psi}{\partial x^{2}}+F^{2} \frac{\partial^{2} \psi}{\partial z^{2}}+2 S^{2} \frac{\partial^{2} \psi}{\partial z \partial x}=2 u_{x} b_{x}
$$

with $\psi$ a meridional streamfunction related to the ageostrophic circulation (i.e. defined as $v_{a}=-\frac{\partial \psi}{\partial z}, w=\frac{\partial \psi}{\partial x}$, with $v_{a}$ the ageostrophic component of the meridional component of the horizontal velocity field. $N^{2}=N^{2}(x, z), S^{2}=-b_{x}$ and $F^{2}=f\left(f+\frac{\partial v}{\partial x}\right)$, where $\frac{\partial v}{\partial x}$ is the relative vorticity associated with the front ${ }^{3}$.

Compared with the QG version (equation 3 in Methods) rewritten in the form of the ageostrophic stream function, equation (2) involves $F^{2}=f\left(f+\frac{\partial v}{\partial x}\right)$ instead of $f^{2}$ as well as $N^{2}(x, z)$ instead of $N^{2}(z)$. In addition, equation (2) involves the extra term $2 S^{2} \frac{\partial^{2} \psi}{\partial z \partial x}$. A dimensional analysis, using $L$ and $D$ as respectively the horizontal and depth scales of the front, indicates that this extra term is of the order of $R i^{-1}$, with Ri the Richardson number, compared to the other two terms on the LHS ${ }^{2,3}$. Since $R i^{-1}$ is not small but close to one at locations of sharp fronts, this term should not be ignored. Nevertheless, a real solution for equation (2) exists if and only if $F^{2} N^{2}-S^{4}>0$. This condition can be written as $f^{2} N^{2}\left(1+\mathrm{Ro}^{-} \mathrm{Ri}^{-1}\right)>0$, with Ro the Rossby number. As such, it places a strong constraint on $\mathrm{Ri}^{-1}$, which is postive and has to be smaller than I1+Rol. This leads to a limitation of the steepness of the front and also to a limitation of the importance of the $S^{2}$ term relatively to others.

However, we do not have access to $F^{2}$ and in particular to $\frac{\partial v}{\partial x}$, the along-front relative vorticity, that can be of order $f$. SSH observations have a spatial resolution too low to resolve $\frac{\partial v}{\partial x}$ at these scales. This is why we use the QG version (equation 3 in Methods). Nevertheless, many studies, starting with Hoskins (1982) ${ }^{4}$ and more recently confirmed by Hakim and Keyser $(2001)^{2}$, emphasize that the SE and QG solutions are qualitatively similar when the condition $F^{2} N^{2}-S^{4}>0$ is met. In particular, the SE and QG ageostrophic circulation, and therefore the w-field, have the same sign. What differs is the amplitude of the w-field, which is larger in the SE solution, as well as the shape of the ageostrophic circulation, which is more tilted and oriented parallel to the isopycnals in the SE solution. Thus, the QG omega solution gives the correct sign for w but the magnitude may be underestimated at low Ri.

This underestimation can however be quantified using the analytical solutions for the QG and SE versions of the omega equation derived in Hakim and Keyser $(2001)^{2}$. These authors show that $w_{S A} \sim w_{Q G}\left[\frac{P_{Q G}}{P V_{P E}}\right]^{1 / 2}$ with $w_{S A}$ and $w_{Q G}$ respectively the solution of the QG and SA equations and $P V_{Q G}$ and $P V_{P E}$ respectively the QG potential vorticity and the Ertel potential vorticity. Using the approximations $P V_{Q G} \sim f N^{2}$ and $P V_{P E} \sim f N^{2}\left[1-R i^{-1}\right]$, as detailed by Thomas et al. 2008 ${ }^{3}$, leads to $\left[\frac{P V_{Q G}}{P V_{P E}}\right]^{1 / 2}=\left[\frac{1}{1-R i^{-}}\right]^{1 / 2}$. When $R i=2$, which corresponds to the strongest fronts in the seal measurements (see main text), we get $w_{S A} \sim 1.4 \times w_{Q G}$. In other words, the maximum $w$-values found in our study should be closer to $140 \mathrm{~m} /$ day than to 100 $\mathrm{m} /$ day. 


\section{Dominance of frontogenesis versus frontolysis understood via the direct cascade of po- tential energy}

The classical frontogenesis process ${ }^{5}$ emphasizes that the increase tendency of a buoyancy gradient embedded in a strong strain field is balanced by a vertical velocity field that tends to decrease this buoyancy gradient (black arrows in Fig. 3b). The reason is that a buoyancy gradient increase destroys the thermal wind balance and this balance is restored by the emergence of the vertical velocity field. These mechanisms lead to the omega equation (see Methods section "Vertical velocities"). As illustrated in Fig. 3b, the frontogenesis process leads to an upward vertical heat flux (red arrows in Fig. 3b) because of the positive correlation between temperature and vertical velocities anomalies.

On the other hand, the frontolysis process, or the destruction of front, occurs when the strain rate decreases and/or changes its direction. As a consequence, the buoyancy gradient relaxes, which is then balanced by a vertical velocity field (and therefore a vertical heat flux) in the opposite direction for the same reason as before (thermal wind balance). The frontolysis process is also captured by the omega equation and is associated with a downward vertical heat flux because of the negative correlation between temperature and vertical velocities anomalies (Fig. 5c). Frontogenesis (frontolysis) processes explain occurrences of positive (negative) vertical heat fluxes displayed in Fig. 5c and Extended Data Fig. 5b.

However, a well-known property of mesoscale eddy turbulence is the direct cascade of potential energy driven by the background strain field. The direct cascade refers to the creation of buoyancy anomalies at smaller and smaller scales and thus to the continuous production of submesoscale fronts ${ }^{6}$. This is why frontogenesis processes statistically dominate frontolysis ones, as can be seen in Fig. S2c where positive buoyancy fluxes are more numerous and of greater magnitude than negative ones. As a consequence, we propose that the dominance of positive vertical heat fluxes at submesoscale as demonstrated in the main text is consistent with the strong background eddy field (Fig. 5f).

\section{Numerical simulation comparison}

The vertical velocity field and vertical heat transport (VHT) calculated from the observations are compared to the same fields obtained from a high-resolution numerical simulation in the Kerguelen area in late spring-early summer (November 2011) performed with the Massachusetts Institute of Technology general circulation model (MITgcm). This high-resolution simulation has a horizontal resolution of $1 / 48^{\circ}, 90$ vertical levels and 10 minute outputs and is described in many papers (see for example Su et al. $\left.(2018)^{7}\right)$. The model domain is $45^{\circ} \mathrm{S}-55^{\circ} \mathrm{S} / 85^{\circ} \mathrm{E}-95^{\circ} \mathrm{E}($ or $\sim 1000 \times 1000 \mathrm{~km})$. This domain is embedded in the MITgcm LLC 4320 global numerical simulation performed at the same resolution but with hourly outputs ${ }^{7}$. Boundary conditions and forcings are supplied by the global simulation.

Vertical sections of daily averaged vertical velocities and vertical heat transport obtained from this simulation are presented in Extended Data Fig. 5. At depths of 100-600 m, intense vertical velocities with a width of 5-10 km are present below the mixed layer and reach $300 \mathrm{~m} /$ day. Similar features are observed for vertical heat transport with values locally reaching $4000 \mathrm{~W} / \mathrm{m}^{2}$. Two-dimensional slices (x,y) of vertical heat transport averaged over 10 days are shown in Extended Data Fig. 6 at 50 and 200 $\mathrm{m}$. This Fig. clearly highlights that the organization of VHT is driven by the background mesoscale strain field, intensified on the eddy edges. This Fig. also shows the dominance of positive VHT in the domain as well as its intensification at depth. Finally, Extended Data Fig. 7 presents the domain-averaged VHT over one month, which reveals similar - although larger values than the ones derived from the observational data. Overall, the similar shape, distribution within the water column, and magnitude of both quantities in the model and in the observations strengthen the results presented in this study, and further highlight the impact of deep reaching ocean fronts on oceanic heat transport.

\section{References}

1. Vallis, G. K. Atmospheric and oceanic fluid dynamics (Cambridge University Press, 2017).

2. Hakim, G. \& Keyser, D. Canonical frontal circulation patterns in terms of green's functions for the sawyer-eliassen equation. Q. J. Royal Meteorol. Soc. 127, 1795-1814 (2001).

3. Thomas, L. N., Tandon, A. \& Mahadevan, A. Submesoscale processes and dynamics. Ocean. modeling an Eddying Regime 177, 17-38 (2008).

4. Hoskins, B. J. The mathematical theory of frontogenesis. Annu. review fluid mechanics 14, 131-151 (1982).

5. Hoskins, B. J. \& Bretherton, F. P. Atmospheric frontogenesis models: Mathematical formulation and solution. J. Atmospheric Sci. 29, 11-27 (1972). 
6. Salmon, R. Baroclinic instability and geostrophic turbulence. Geophys. \& Astrophys. Fluid Dyn. 15, 167-211 (1980).

7. Su, Z., Wang, J., Klein, P., Thompson, A. F. \& Menemenlis, D. Ocean submesoscales as a key component of the global heat budget. Nat. communications $\mathbf{9 ,} 775$ (2018).
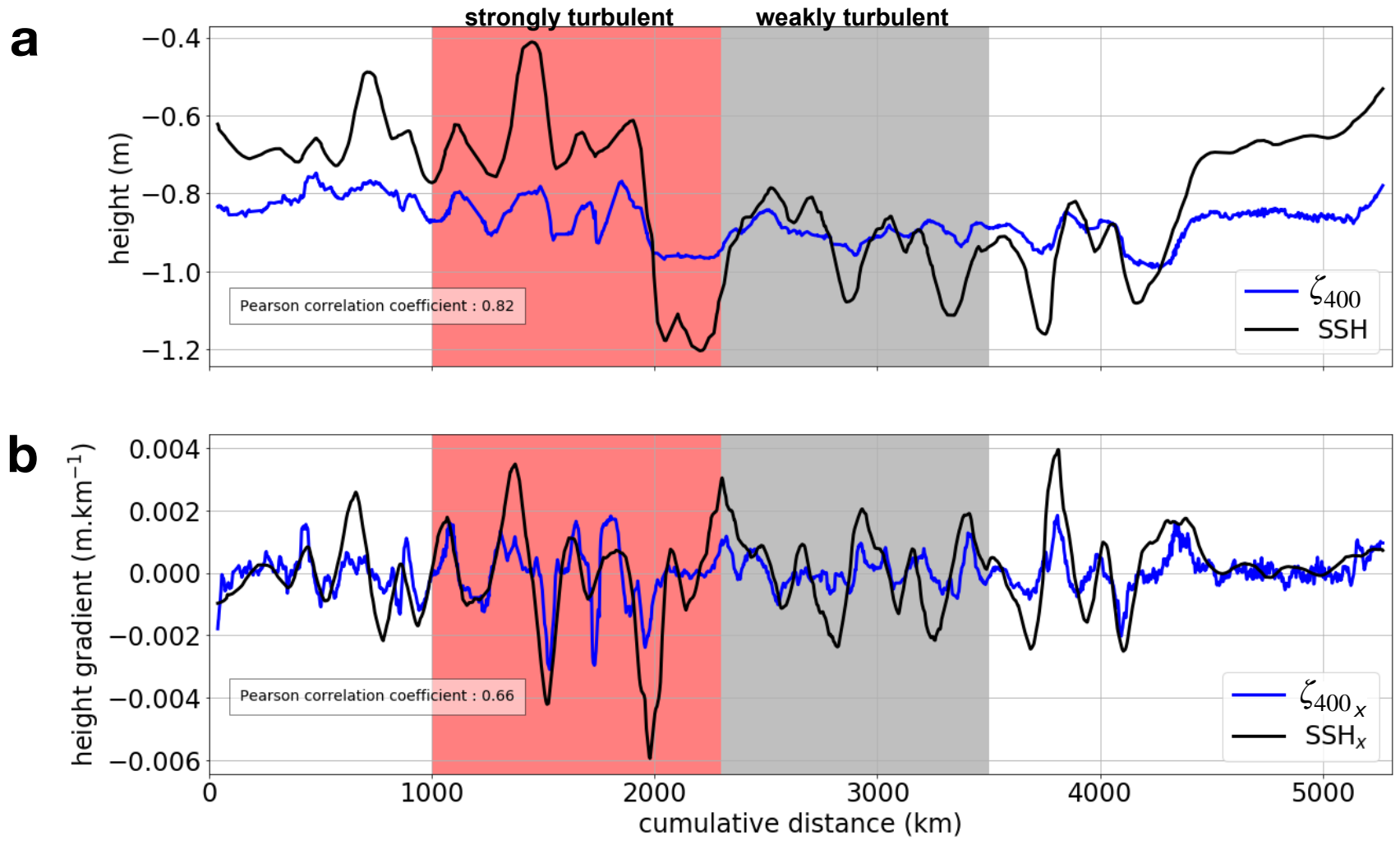

Supplementary Fig. S1. SSH and steric height $\left(\zeta_{400}\right)$. a) Time series of satellite SSH (black line) along the seal's path and steric height ( $\zeta_{400}$, blue line) calculated from the SES dataset down to $400 \mathrm{~m}$. b) Time series of the lateral gradients of SSH ( $\mathrm{SSH}_{x}$, black line) and steric height $\left(\zeta_{400_{x}}\right.$, blue line). On both panels, the red (grey) zone corresponds to the strongly (weakly) turbulent area of Fig. 2(S1i). 

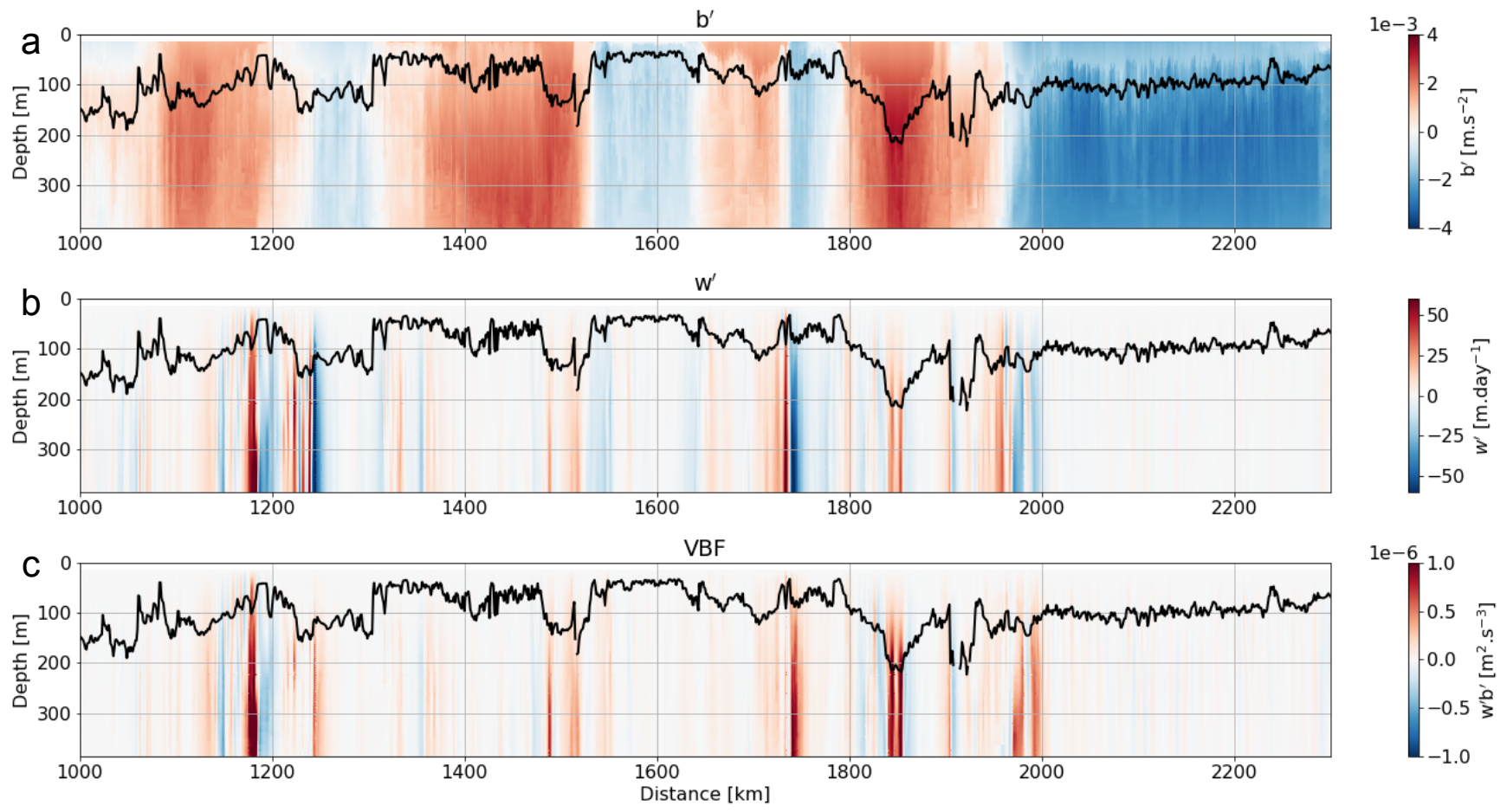

Supplementary Fig. S2. Buoyancy and temperature anomalies and vertical buoyancy fluxes from seal and satellite data Same as Fig. 5 but for buoyancy. a) Vertical section of buoyancy anomaly sampled by the seal. b) Vertical section of vertical velocity anomaly derived from the seal and satellite data by solving the omega equation (see main text and Methods). c) Vertical section of vertical buoyancy flux (or transport, VBF) defined as $w^{\prime} b^{\prime}$, with $w^{\prime}$ and $b^{\prime}$ are the anomalies of vertical velocity and buoyancy, respectively. The mixed layer depth is shown in bold black. From panel c) it is clear that frontogenesis (positive VBF, in red) dominates frontolysis (negative VBF, in blue). 